Louisiana State University

LSU Digital Commons

$9-17-2020$

Effect of Oxidation Level on the Interfacial Water at the Graphene Oxide-Water Interface: From Spectroscopic Signatures to Hydrogen-Bonding Environment

Rolf David

Aashish Tuladhar

Le Zhang

Christopher Arges

Revati Kumar

Follow this and additional works at: https://digitalcommons.Isu.edu/chemistry_pubs

Part of the Physical Chemistry Commons 


\section{Effect of Oxidation Level on the Interfacial Water at the Graphene Oxide-Water Interface: From Spectroscopic Signatures to Hydrogen-Bonding Environment}

Published as part of The Journal of Physical Chemistry virtual special issue "Lawrence R. Pratt Festschrift". Rolf David, Aashish Tuladhar,* Le Zhang, Christopher Arges, and Revati Kumar*

Cite This: J. Phys. Chem. B 2020, 124, 8167-8178

Read Online

ABSTRACT: The interfacial region of the graphene oxide (GO)-water system is nonhomogenous due to the presence of two distinct domains: an oxygen-rich surface and a graphene-like region. The experimental vibrational sum-frequency generation (vSFG) spectra are distinctly different for the fully oxidized GO-water interface as compared to the reduced GO-water case. Computational investigations using ab initio molecular dynamics were performed to determine the molecular origins of the different spectroscopic features. The simulations were first validated by comparing the simulated vSFG spectra to those from the experiment, and the contributions to the spectra from different hydrogen bonding environments and interfacial water orientations were determined as a function of the oxidation level of the GO sheet. The ab initio simulations also revealed the reactive nature of the GO-water interface.

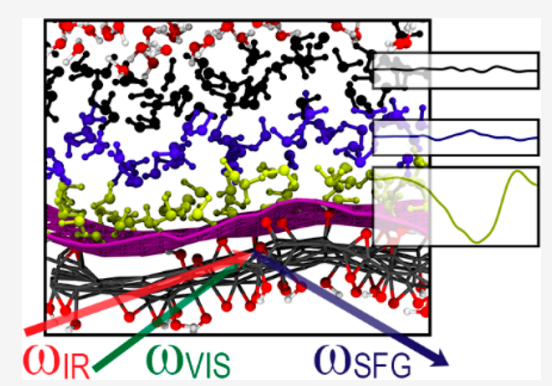

\section{INTRODUCTION}

Graphene oxide (GO), whether a single layer or a few layers of exfoliated sheets from graphite oxide, has recently received a lot of attention in the literature due to a range of potential applications. $^{1-35} \mathrm{GO}$ consists of graphene sheets with oxygenated groups, and a number of studies have revealed a wide range of oxygen functional groups, such as hydroxyls and epoxides, ${ }^{11}$ carboxylic acids, or sulfonates groups, ${ }^{6,36}$ on these sheets as well as how these groups are arranged on the surface. ${ }^{37-41}$ A key question that arises is how, depending on their number and partitioning, these oxygen functional groups can favor or prohibit reactions at the GO-liquid interface in aqueous media. To probe interfaces, several surface-specific techniques can be used such as environmental scanning electron microscopy (ESEM), secondary ion mass spectrometry (SIMS), Auger electron spectroscopy (AES), etc. ${ }^{42-46}$ One method, vibrational sum-frequency generation (vSFG) ${ }^{47,48}$ has received a lot of attention for characterizing interfaces experimentally ${ }^{49-57}$ and in conjunction with simulations. ${ }^{51,52,58-62}$ The synergy between vSFG experiments and molecular simulations allows for an in-depth probing of the interface, permitting a finer molecular interpretation of the underlying interfacial region. In this paper, an analysis of the graphene-oxide-water interface by ab initio molecular dynamics (AIMD) at different levels of oxidation was performed to provide insight on the effect of the different structural domains of graphene-oxide (organic, aromatic rich regions vs oxygenrich hydrophilic regions) on the interfacial water structure. Furthermore, the effect of the oxidation level of the GO sheet on the water structure was also studied. These results are put into perspective with the experimental vSFG spectra of these systems as a function of oxidation level, thereby not just confirming the accuracy of said ab-initio methods but also providing insight into the molecular origins of the spectral signatures in the experimental vSFG spectra. This paper is divided into four sections. Both the computational and experimental methods are outlined in Section II, the results are described and discussed in Section III, and the conclusions are presented in Section IV.

\section{MATERIALS AND METHODS}

II.a. Ab Initio MD Setup. The graphene oxide sheets in this study consist of a single layer composed of 180 carbon atoms (to have an $\sim 22.0 \times 21.2 \AA$ graphene sheet) and a varying number of oxygen functional groups. The $\mathrm{GO}_{4 / 1}$ and $\mathrm{GO}_{2 / 1}$ sheets were constructed based on the work of Sinclair et al. ${ }^{41}$ The former consists of 24 epoxide groups and 20 hydroxyl groups for a ratio $\mathrm{C} / \mathrm{O}$ of 4.09 for the former, while 50 epoxide groups and 40 hydroxyl groups were introduced for a ratio of $\mathrm{C} / \mathrm{O}$ of 2.00 for the latter case. The GO/water interface was generated using the packmol software ${ }^{63}$ by adding 265 water

Received: June 10, 2020

Revised: August 10, 2020

Published: August 17, 2020 


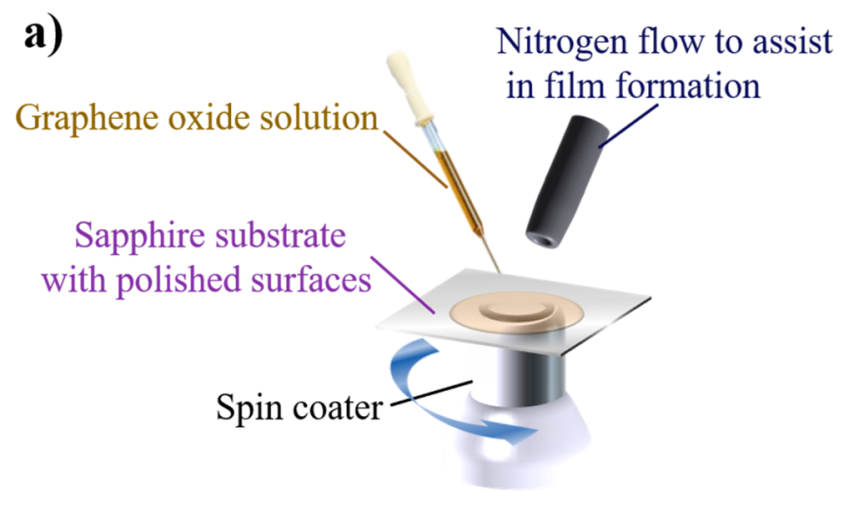

b) Thermal reduction under nitrogen

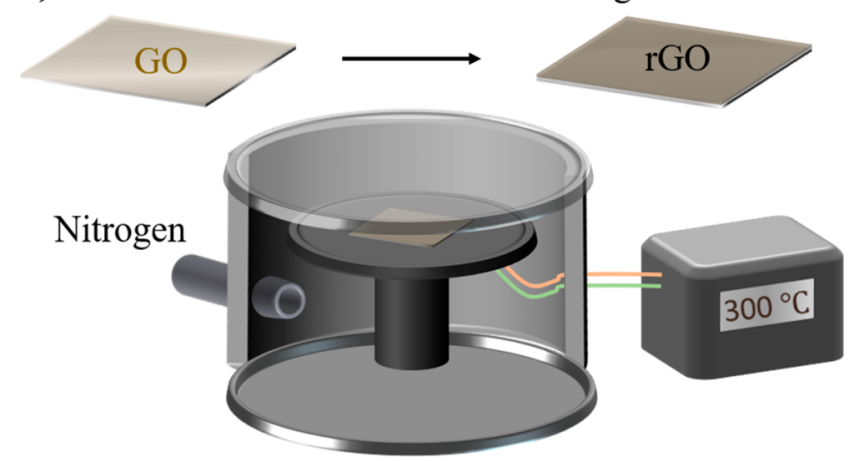

Figure 1. (a) Spin-coating process for preparing thin films of GO on transparent sapphire substrates with a polished flat surface. (b) Thermal reduction of GO thin films to $\mathrm{rGO}$ in a thermal annealing chamber under nitrogen environment.

molecules on one side of the GO sheet generating a solvent layer having a thickness of $20 \AA$. With the moltemplate software, ${ }^{64}$ parameter files for both systems were created using the OPLS-AA ${ }^{65}$ force field for the GO sheet along with the $\mathrm{SPC} / \mathrm{E}^{66}$ force field for water. A $70 \AA$ thick layer of vacuum is added in the $z$-direction (direction perpendicular to the interface) for both sets of systems resulting in a box of dimensions $22.0 \times 21.2 \times 104.0 \AA$ A. All simulations were performed using periodic boundary conditions (PBC). For the classical molecular dynamics (MD) simulations, long-range electrostatic interactions were evaluated using the particleparticle particle-mesh (PPPM) ${ }^{67}$ method based on the Ewald summation method with a cutoff of $12.0 \AA$, while the LennardJones interactions used a simple cutoff at $12.0 \AA$. All water bonds and angles were constrained using the SHAKE algorithm. ${ }^{68}$ An initial geometry minimization was performed followed by a 500 ps equilibration run (with a time step of 0.5 fs) in the NVT ensemble (where $\mathrm{N}$ indicates the number of particles in the system, $\mathrm{V}$ indicates the system volume, and $\mathrm{T}$ indicates the absolute temperature of the system) with the temperature set to $300 \mathrm{~K}$ with a Nosé-Hoover thermostat ${ }^{69,70}$ and a time constant of $50 \mathrm{fs}^{-1}$. A production run was performed in the same ensemble for $1 \mathrm{~ns}$ with a time step of $0.5 \mathrm{fs}$. Snapshots were extracted every $200 \mathrm{ps}$, resulting in five snapshots for each system. For each snapshot, and for both systems, geometry optimization and cell relaxation were done using the $\mathrm{CP} 2 \mathrm{~K}^{71,72}$ program with the L-BFGS algorithm. ${ }^{73}$ The force evaluations were done at the density functional theory (DFT) level with the revPBE ${ }^{74,75}$ functional and the empirical D3 ${ }^{76}$ dispersion, with a DZVP-MOLOPT-SR ${ }^{77}$ basis set and GTH pseudopotentials. ${ }^{78-80}$ The $c$ cell parameter was kept fixed at $70.0 \AA$, giving a vacuum layer $40.0 \AA$ thick, and PBC were applied in all directions using a periodic Poisson solver for electrostatics. For each optimized geometry, a 25 ps long NVT simulation at $300 \mathrm{~K}$ with a time step of $0.5 \mathrm{fs}$ was then performed with the canonical sampling through a velocity rescaling (CSVR) thermostat ${ }^{81}$ and a $100 \mathrm{fs}^{-1}$ time constant. The position and velocities were extracted every $1 \mathrm{fs}$, and the first 5 ps of each trajectory were discarded as equilibration. The total sampling for both the $\mathrm{GO}_{2 / 1}$ and $\mathrm{GO}_{4 / 1}$ case was 100 ps $(5 \times 20$ ps $)$.

II.b. Preparation of the Graphene Oxide Samples. A large-area oligo-layered GO flake solution having a concentration of $5 \mathrm{mg} \mathrm{mL}^{-1}$ was purchased from NewMater Nanotechnology Co. Ltd. Transparent sapphire circular disks with flat surfaces (surface roughness $<1.0 \mathrm{~mm}$ ) were acquired from Meller Optics, Inc. These disks had an area of $20.27 \mathrm{~cm}^{2}$ (diameter of $5.08 \mathrm{~cm}$ ) and a thickness of $0.33 \mathrm{~nm}$. To attain a conformal graphene flake layer onto the substrate, a diluted solution of GO flakes was prepared by mixing the $5 \mathrm{mg} \mathrm{mL}^{-1}$ graphene oxide with methanol and water with a weight ratio of 1:1740:100 (graphene oxide/methanol/water). The dilute solution was spin-coated onto the transparent sapphire substrate at $3000 \mathrm{rpm}$ for $45 \mathrm{~s}$. Prior to the deposition of the solution on the substrate for spin coating, 1 psi of nitrogen flow was applied to the surface of the sapphire substrate from nearly normal incidence. Two more identical samples were prepared, and these samples were treated thermally to reduce graphene oxide on the sapphire substrate. The thermal reduction of graphene oxide thin film was conducted at 300 ${ }^{\circ} \mathrm{C}$ in a nitrogen-filled chamber for $10 \mathrm{~min}$ for one sample and $6 \mathrm{~h}$ for another sample. The successful reduction of the GO film was evident by the film color change and the vSFG results (vide supra). Figure 1 depicts the scheme to prepare thin GO/ rGO (where rGO indicates reduced graphene oxide) films on transparent sapphire substrates.

II.c. Experimental vSFG Setup. A picosecond scanning vibrational sum frequency generation spectrometer (EKSPLA), which has been described in previous works, ${ }^{82,83}$ was used to perform the vSFG experiments. Briefly, the vSFG spectrometer is a commercial setup that uses a $532.1 \mathrm{~nm}$ visible beam and a tunable infrared beam overlapped spatially and temporally at the sample surface. The angle of incidence is $35^{\circ}$ and $31^{\circ}$ for the visible and the IR beams, respectively. The spatial resolution of the setup is $\sim 6 \mathrm{~cm}^{-1}$. An SSP polarization geometry (where S, S, and P refer to the polarization of sum frequency, visible, and IR photons, respectively) was used. In all experiments, each scan was obtained with an increment of 8 $\mathrm{cm}^{-1}$ and an average over 300 laser shots per point. The energy of the visible beam is typically $\sim 200 \mu \mathrm{J}$, and that of the IR beam is $\sim 180 \mu \mathrm{J}$. The SFG photons were detected using a high-gain low-noise photomultiplier (Hamamatsu R585), which is integrated into the Ekspla system. The voltage of the photomultiplier tube (PMT) was set at $1400 \mathrm{~V}$. The vSFG signal is normalized with respect to the visible and IR pulse energy.

The graphene oxide film grown on an alumina substrate was placed in a precleaned (thorough rinsing with detergent and a copious amount of ion-exchanged nanopure deionized water followed by drying with compressed $\mathrm{N}_{2}$ and finally UV/ozone cleaned (Novascan Technologies) for $15 \mathrm{~min}$ ) home-built Teflon sample cell that was designed to allow for the 

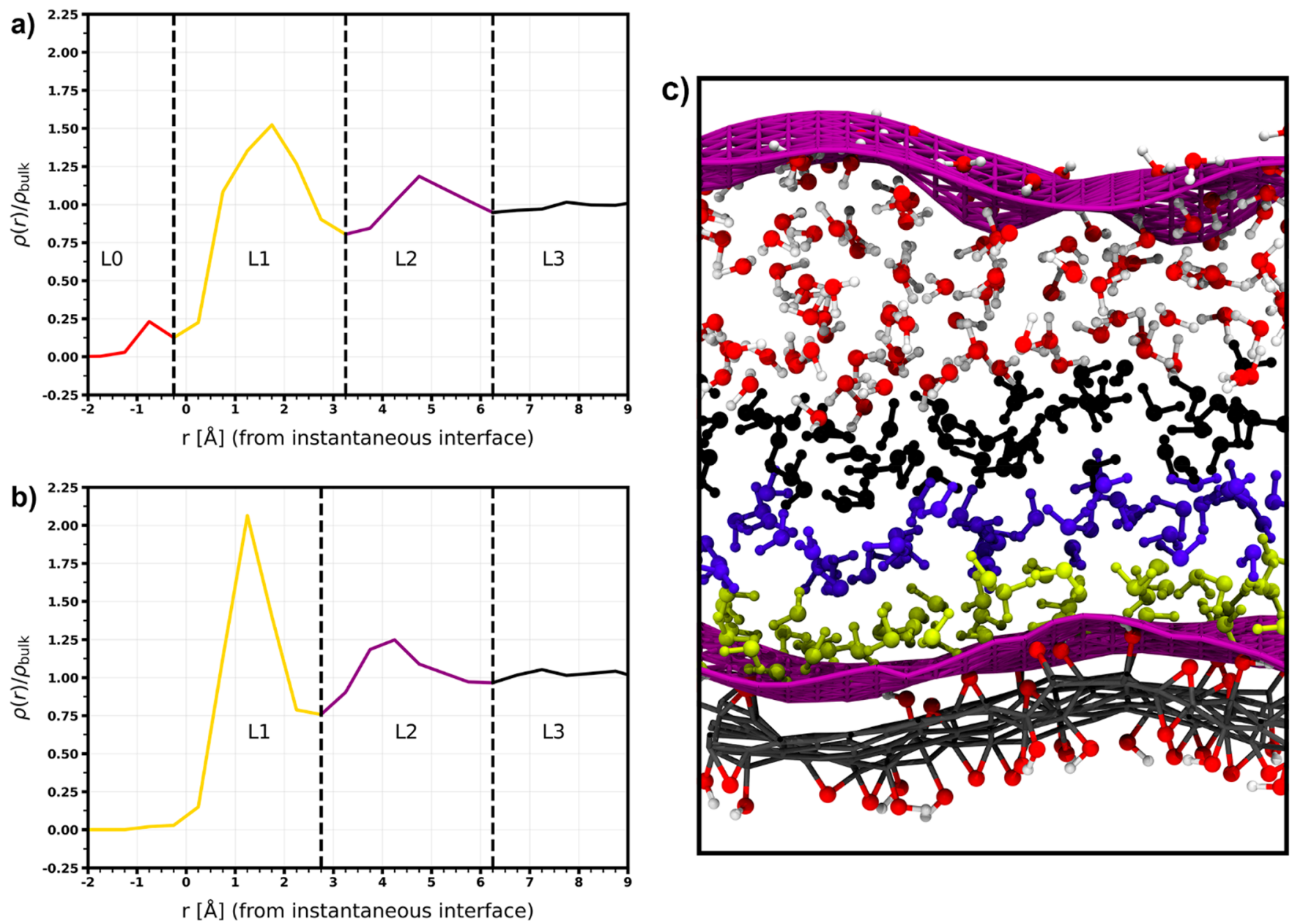

Figure 2. Ratio of the density of water over the bulk density of water as a function of the distance to the instantaneous water interface for $(\mathrm{a}) \mathrm{GO}_{2 / 1}$ and (b) $\mathrm{GO}_{4 / 1}$. (c) Representation of the $\mathrm{GO}_{4 / 1}$ system with the instantaneous water interfaces (in purple), the GO sheet, and the water layers. Water layers are yellow, purple, and black, representing L1, L2, and L3, respectively. Carbon is in gray; for waters beyond the L3 layer, the oxygen atoms are in red, and the hydrogen atoms are in white.

introduction of an aqueous solution without moving the sample surface. Laboratory-equilibrated deionized water $(\mathrm{pH}$ $\approx 6$ ) was used for the experiments. The schematic experimental geometry is shown in Scheme $S 1$ in the Supporting Information.

II.d. Surface-Specific Velocity-Velocity Correlation Function from Simulations. In this study, the surfacespecific velocity-velocity correlation function (ssVVCF) formalism proposed by Otho et al. ${ }^{84}$ was used. The method is described in detail in ref 84 , and here just a brief description of the method is presented. In the case of just the IR spectrum, the IR response function (from the fluctuation-dissipation theorem) is related to the time derivative dipole-dipole correlation function. ${ }^{85}$ The molecular dipole moment in turn is related to the permanent dipole moment of the molecule and the transition dipole moment of the normal mode. The latter is determined by multiplying the transition dipole by the normal mode vector in the molecular frame. Finally, the molecular dipole moment is converted to the lab frame through a rotational matrix that is applied to both the permanent and transition dipole moments in the molecular frame. The $\mathrm{O}-\mathrm{H}$ stretch response is the one under consideration and can be considered to be decoupled from librational motion. The latter is dominated by the dynamics of the permanent dipole moment, while the $\mathrm{O}-\mathrm{H}$ stretch response is dominated by the transition dipole moment. Since the main contribution to the $\mathrm{O}-\mathrm{H}$ stretch normal mode is the $\mathrm{O}-\mathrm{H}$ vector, ${ }^{86}$ the normal mode vector in the laboratory frame can be replaced by the bond vector resulting in a simplified description for the IR response to the $\mathrm{O}-\mathrm{H}$ stretch that is proportional to the $\mathrm{O}-\mathrm{H}$ stretch velocity autocorrelation function. A similar reasoning was applied by Otho et al. for the SFG response function (which now also includes the polarizability tensor), connecting both the IR and SFG response to essentially different velocityvelocity type correlation functions.

$\chi_{\mathrm{xxz}}^{(2), \mathrm{R}}(\omega)$ is the resonant component of the second-order susceptibility (where $z$ is the direction perpendicular to the interface) and can be written as

$$
\chi_{x x z}^{(2), \mathrm{R}}(\omega)=\frac{Q(\omega) \mu(\omega) \alpha(\omega)}{i \omega^{2}} \chi_{x x z}^{\mathrm{ssVCF}}(\omega)
$$

where $Q(\omega)$ is the harmonic quantum-correction factor ${ }^{87}$ and is given by

$$
Q(\omega)=\frac{\beta \hbar \omega}{1-\mathrm{e}^{-\beta \hbar \omega}}
$$

with $\beta=\frac{1}{k T}$ and $T$ is the temperature set to $300 \mathrm{~K}$. $\hbar$ is the reduced Planck's constant and $k$ is the Boltzmann constant. 
The non-Condon effects were taken into account by the frequency-dependent transition dipole moment and the frequency-dependent transition polarizability $(\mu(\omega)$ and $\alpha(\omega)$, respectively) parametrized in the work of Corcelli and Skinner ${ }^{88,89}$

$$
\begin{aligned}
& \mu(\omega)=\left(1.377+\frac{53.03(3737.0-\omega)}{6932.2}\right) \mu^{0} \\
& \alpha(\omega)=\left(1.271+\frac{6.287(3737.0-\omega)}{6932.2}\right) \alpha^{0}
\end{aligned}
$$

where $\omega$, in (3) and (4), is specifically expressed in $\mathrm{cm}^{-1}$.

Finally, $\chi_{\mathrm{xxz}}^{\mathrm{ssVCF}}(\omega)$ is given by

$$
\begin{aligned}
\chi_{x x z}^{\mathrm{ssVCF}}(\omega)= & \int_{0}^{\infty} \mathrm{d} t \mathrm{e}^{-i \omega t}\left\langle\sum_{i, j} g\left(r_{i j}(0)\right) r_{z, i}^{\cdot \mathrm{OH}}(0)\right. \\
& \left.\frac{\dot{\vec{r}}_{j}^{\mathrm{OH}}(t) \cdot \vec{r}_{j}^{\mathrm{OH}}(t)}{\left|\vec{r}_{j}^{\mathrm{OH}}(t)\right|}\right\rangle
\end{aligned}
$$

where $i$ and $j$ are the $i$ th and $j$ th oscillators, respectively. $\dot{r}_{\mathrm{z}, \mathrm{i}}^{\mathrm{OH}}$ is the $z$ component of the velocity of the ith oscillator, and $\dot{\vec{r}}_{j} \mathrm{OH}$ and $\vec{r}_{j} \mathrm{OH}$ are, respectively, the velocity vector and the displacement vector of the $j$ th oscillator. $r_{i j}$ is the distance between the ith center of mass and the $j$ th center of mass of the respective oscillators, and $g(r)$ is a switching function:

$$
g\left(r_{i j}\right)=\left\{\begin{array}{cc}
1, & r_{i j} \leq 2.0 \AA \\
0, & r_{i j}>2.0 \AA
\end{array}\right.
$$

This switching function controls the cross-correlation terms between two oscillators: an $r_{i j}$ cutoff at $2.0 \AA$ ensures only intramolecular coupling terms. The time correlation was evaluated for a $t_{\max }$ of 10 ps. A smoothing Hann window function, $f(t)$, was applied to the Fourier transform of the time correlation function.

$$
f(t)=\left\{\begin{array}{cl}
\cos ^{2}\left(\frac{\pi t}{2 \tau}\right), & 0<t<\tau \\
0, & t>\tau
\end{array}\right.
$$

The parameter $\tau$ was set to 0.50 ps.

Additional details including other switching functions that were used are given in the Supporting Information.

\section{RESULTS AND DISCUSSION}

III.a. Average Water Density Fluctuations from the Instantaneous Water Interface. To characterize the interface between the GO sheet and the water, the WillardChandler instantaneous interface ${ }^{90}$ was employed, as it provides a robust definition of the interfacial region. The ratio of the water density to the bulk density of water as a function of the distance to the instantaneous water interface is reported in Figure 2. Well-defined water layers, based on the minima in the density distributions in Figure 2, can be seen. This type of layering has also been seen for water next to other solid interfaces in studies performed by Gaigeot et al. ${ }^{91,92}$ In the $\mathrm{GO}_{4 / 1}$ case three distinct layers of water, namely, L1, L2, and L3, with increasing distance from the instantaneous interface are seen. A fourth layer, L0, is only present in the case of $\mathrm{GO}_{2 / 1}$, in the negative distance region (on the other side of the instantaneous interface) and corresponds to a small number of waters "trapped" on the GO sheet by the oxygen functional groups. Between the $\mathrm{GO}_{2 / 1}$ and $\mathrm{GO}_{4 / 1}$, the major difference for the density resides in the $\mathrm{L} 1$ layer, which is more structured for $\mathrm{GO}_{4 / 1}$ due to a sharper peak, as well as the presence of an L0 layer solely in the case of $\mathrm{GO}_{2 / 1}$.

III.b. Hydrogen Bond Analysis of the Interfacial Waters. The hydrogen bond network of the waters was analyzed for both cases. A naming scheme for the different hydrogen bonding classes of water based on the work of Skinner et al. ${ }^{93}$ was used here. A water is defined as residing in a hydrogen-bonding class $\mathbf{N}_{a}$, where $\mathbf{N}$ is the total number of hydrogen bonds (see Scheme S2 for definition of a hydrogen bond) a water molecule is involved in, and the subscript a refers to the number of hydrogen bonds involving the $\mathrm{H}$ atoms of the water under consideration: $\mathbf{a}$ is $\mathbf{S}$ for a single donor water, $\mathbf{D}$ is for a double donor water, and $\mathbf{T}$ and $\mathbf{Q}$ are for triple and quadruple donor waters, respectively. Water-Water hydrogen bonds are considered as well as water-oxygenbearing groups hydrogen bonds. Figure 3 shows the percentage of hydrogen-bonding classes for all water within the L0 (Figure $3 a$ ) and $\mathrm{L} 1$ (Figure 3b) layers for $\mathrm{GO}_{2 / 1}$ and the $\mathrm{L} 1$ layer for $\mathrm{GO}_{4 / 1}$. The composition of the L0 layer is very different from
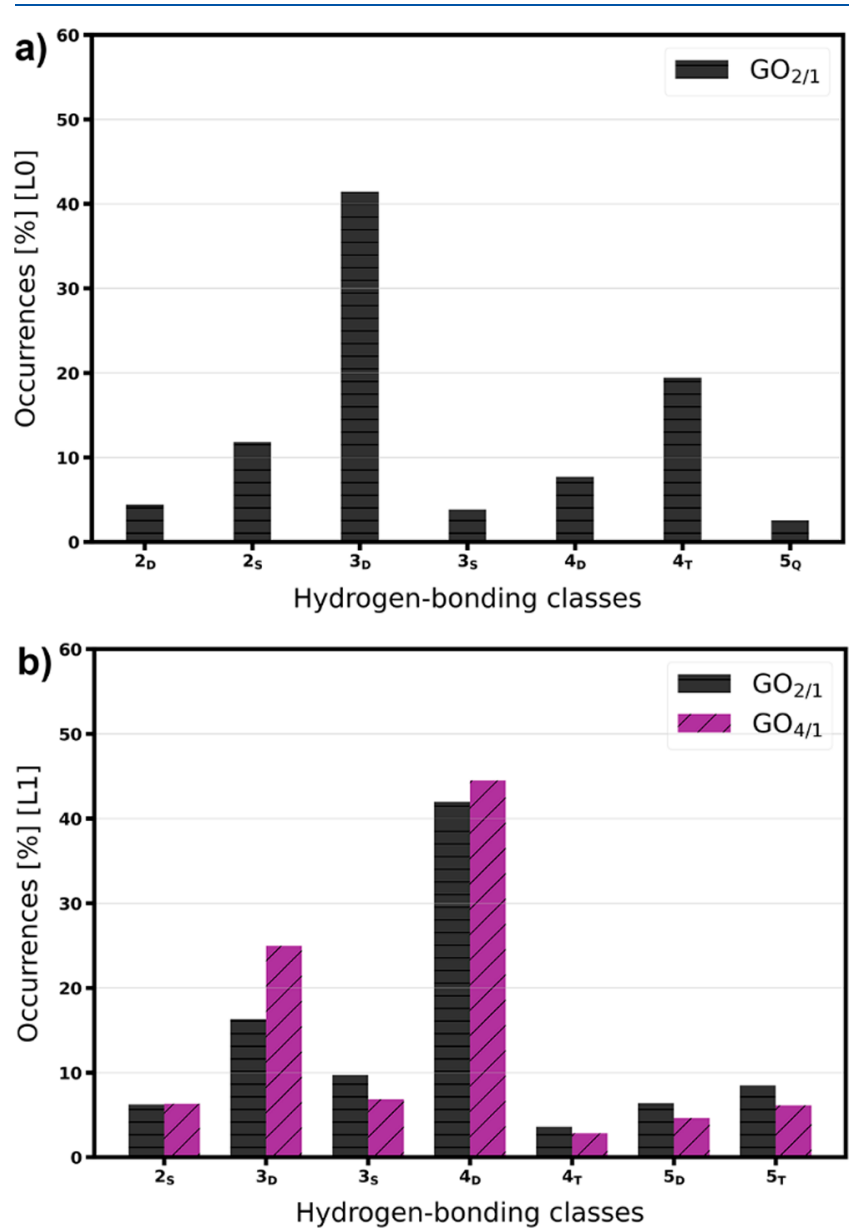

Figure 3. Distribution (in percentage) of the different hydrogenbonding classes of water. (a) In the $\mathrm{L} 0$ layer for $\mathrm{GO}_{2 / 1}$ (in black, horizontal stripes). (b) In the $\mathrm{L} 1$ layers for $\mathrm{GO}_{2 / 1}$ (in black, horizontal stripes) and $\mathrm{GO}_{4 / 1}$ (purple, slanted stripes). 

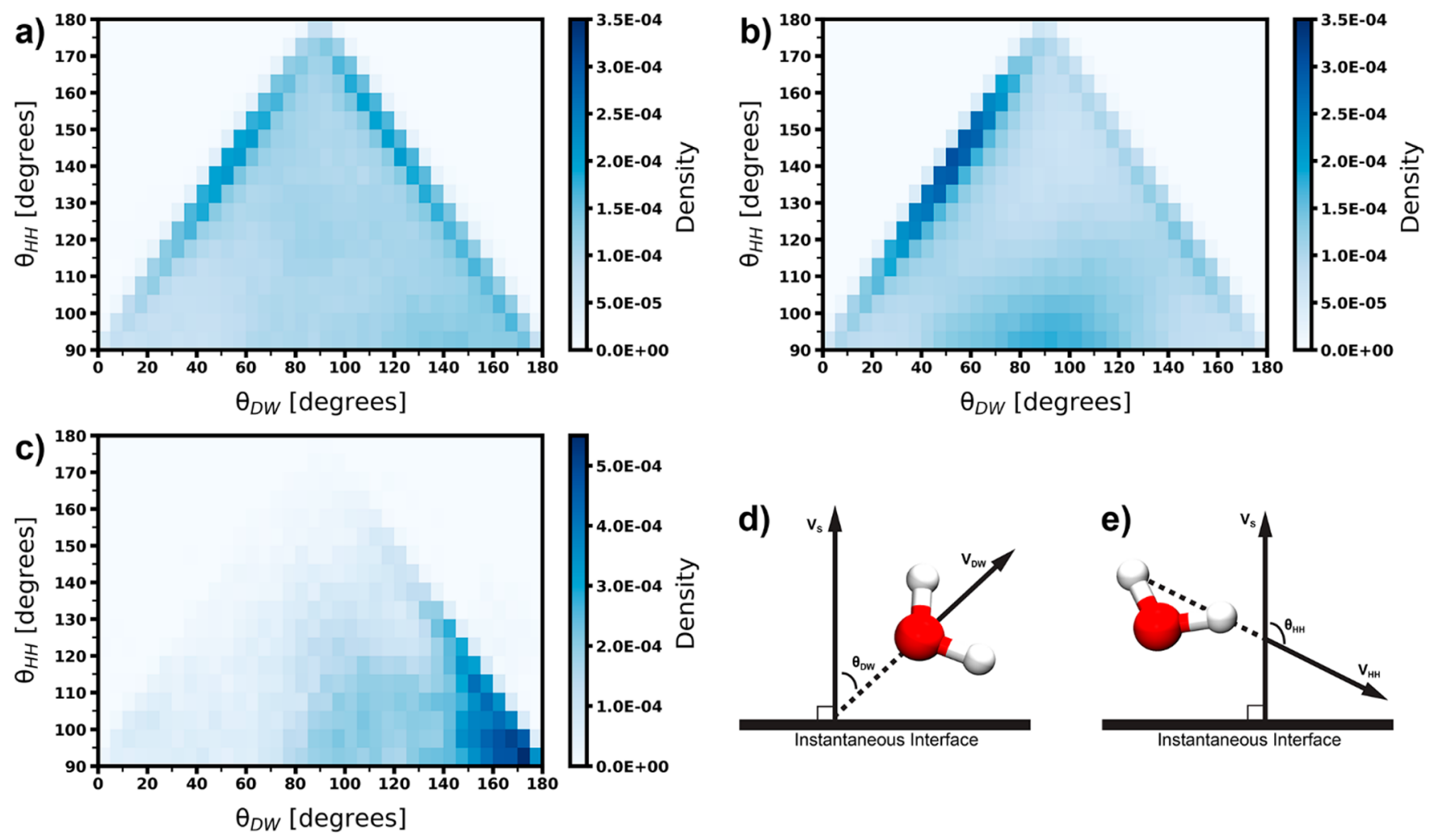

Figure 4. Two-dimensional histograms of the joint distribution of the $\theta_{\mathrm{DW}}$ angle and the $\theta_{\mathrm{HH}}$ angle for (a) $\mathrm{GO}_{2 / 1} \mathrm{~L} 1,(\mathrm{~b}) \mathrm{GO}_{4 / 1} \mathrm{~L} 1$, and $(\mathrm{c}) \mathrm{GO}_{2 / 1}$ L0. (d) Definition of the water orientation angle $\left(\theta_{\mathrm{DW}}\right)$. $\mathbf{V}_{\mathrm{DW}}$ is the water bisector and $\mathbf{V}_{\mathrm{S}}$ is the vector normal to the instantaneous surface (always pointing in the direction of the water molecule). (e) Definition of the water orientation angle $\left(\theta_{\mathrm{HH}}\right) . \mathbf{V}_{\mathrm{HH}}$ is the vector connecting the two hydrogen atoms of a water molecule vector, and $\mathbf{V}_{S}$ is the vector normal to the instantaneous water surface (always pointing in the direction of the water molecule). All distributions were normalized to have unit area.

that of the L1 layer, with the major class in L1 being $4_{D}$, while $\mathbf{3}_{\mathrm{D}}$ is the dominant hydrogen-bonding configuration of waters in LO.

Although double donors are the most common in both L0 and L1, the waters in L0 tend to accept fewer hydrogen bonds. This point is reinforced by the second major class present in L0, namely, $4_{\mathrm{T}}$, which like $3_{\mathrm{D}}$ has only one acceptor hydrogen bond (the same goes for $\mathbf{2}_{\mathrm{S}}$ and $\mathbf{5}_{\mathrm{Q}}$ ). This can be explained by a specific orientation of the water molecule in this L0 layer, where a water oxygen is less readily accessible to other waters (or hydroxyl groups) to accept hydrogen bonds, but its hydrogens are available to donate hydrogen, a point that will be discussed further. Additionally, in this layer $\sim 92 \%$ of the water molecules present are engaged in a hydrogen bond with an oxygen-bearing group of the GO: this is due to a higher number of oxygenated defects and due to the "trapped" position of the water. Approximately $44 \%$ of these waters are both donating and accepting from an oxygen-bearing group.

For the $\mathrm{L} 1$ layer, compared to $\mathrm{GO}_{4 / 1}, \mathrm{GO}_{2 / 1}$ tends to have $\mathrm{GO}_{2 / 1}$ with fewer $3_{\mathrm{D}}(-8.6 \%)$ and $4_{\mathrm{D}}(-2.5 \%)$ waters compared to $\mathrm{GO}_{4 / 1}$, while having a greater number of $3_{\mathrm{S}}$ $(+2.9 \%)$ waters. Additionally, $\mathrm{GO}_{2 / 1}$ has a larger percentage of waters with, overall, four or a higher number of total hydrogen bonds that are double or triple the donor $\left(4_{\mathrm{T}},+0.8 \%, 5_{\mathrm{D}}\right.$, $+1.8 \%$, and $\left.5_{\mathrm{T}},+2.4 \%\right)$. Additionally, overall one can see an increase of single donors $(+3.3 \%)$ and triple donors $(+4.0 \%)$ at the expense of the double donors $(-9.0 \%)$. Between $\mathrm{GO}_{4 / 1}$ and $\mathrm{GO}_{2 / 1}$ there is an increase in the number of waters engaged in hydrogen bonds (donating or accepting) with an oxygen-bearing group $(+6.4 \%)$, the most increase comes from the waters accepting at least one hydrogen bond from an oxygen-bearing group $(+9.8 \%)$ or both accepting and donating one $(+6.0 \%)$. Compared to the LO layer, in the L1 layer for $\mathrm{GO}_{2 / 1}$, only $8.3 \%$ of the waters are both accepting and donating to an oxygen-bearing group.

To summarize, the L1 layer, for both systems, has $4_{D}$ as the major class, with the second one being $3_{\mathrm{D}}$. $\mathrm{GO}_{2 / 1}$ sees a small decrease in $4_{D}$ and a bigger one in $3_{D}$, but its number of highly hydrogen-bonded waters $\left(\mathbf{4}_{\mathrm{T}}, \mathbf{5}_{\mathrm{D}}, \mathbf{5}_{\mathrm{T}}\right)$ is greater than $\mathrm{GO}_{4 / 1}$, due to an increase of waters engaged in hydrogen bonds (donating or accepting) with an oxygen-bearing group $(+6.4 \%)$.

III.c. Interfacial Water Orientation. To have a better understanding of the orientation of the water molecules around the interface between the graphene oxide sheet and water, the orientation of the water molecules was examined. Figure 4 shows the joint distribution of the $\theta_{\mathrm{DW}} / \theta_{\mathrm{HH}}$ angles for water molecules in layer $\mathrm{L} 1$ for $\mathrm{GO}_{2 / 1}$ (Figure $4 \mathrm{a}$ ) and $\mathrm{GO}_{4 / 1}$ (Figure 4b) and in layer L0 for $\mathrm{GO}_{2 / 1}$ (Figure 4c), as well as the definition of the two relevant angles chosen, $\theta_{\mathrm{DW}}$ (Figure $4 \mathrm{~d}$ ) and $\theta_{\mathrm{HH}}$ (Figure $\left.4 \mathrm{e}\right)$. In the Supporting Information, the distribution for each trajectory is given, and the results are shown to be consistent with the overall distribution (Figures $\mathrm{S} 1$ and S2).

For the $\mathrm{GO}_{2 / 1} \mathrm{~L} 1$ layer, two main orientations are present: one with values ranging from $50^{\circ}$ to $55^{\circ}$ and from $135^{\circ}$ to $140^{\circ}$ for $\theta_{\mathrm{DW}}$ and $\theta_{\mathrm{HH}}$, respectively, corresponding to an orientation shown in Figure $5 \mathrm{a}$, with one hydrogen pointing away from the instantaneous interface. The second orientation ranges from $120^{\circ}$ to $125^{\circ}$ and from $140^{\circ}$ to $145^{\circ}$ for $\theta_{\mathrm{DW}}$ and 


\section{a)}

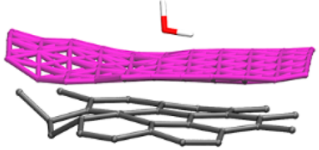

c)

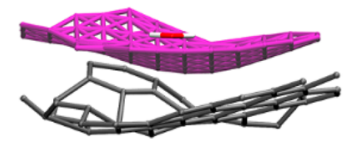

b)

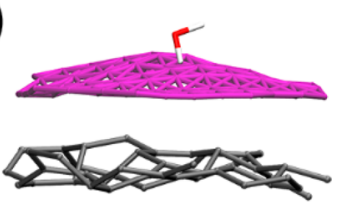

d)

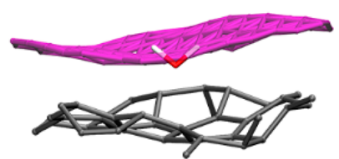

Figure 5. Selective geometries for characteristic $\theta_{\mathrm{DW}} / \theta_{\mathrm{HH}}$ pairs. Only the carbons of the GO sheet are represented in gray for clarity; the instantaneous surface is represented in purple, and the water molecule is represented in red for oxygen and white for hydrogen: (a) $\theta_{\mathrm{DW}} / \theta_{\mathrm{HH}}$ pair values equal to $50^{\circ} / 135^{\circ}\left(\mathrm{GO}_{4 / 1} \mathrm{~L} 1\right.$ and $\left.\mathrm{GO}_{2 / 1} \mathrm{~L} 1\right)$. (b) $\theta_{\mathrm{DW}} /$ $\theta_{\mathrm{HH}}$ pair values equal to $120^{\circ} / 140^{\circ}\left(\mathrm{GO}_{2 / 1} \mathrm{~L} 1\right)$. (c) $\theta_{\mathrm{DW}} / \theta_{\mathrm{HH}}$ pair values equal to $140^{\circ} / 90^{\circ}\left(\mathrm{GO}_{4 / 1} \mathrm{~L} 1\right)$. (d) $\theta_{\mathrm{DW}} / \theta_{\mathrm{HH}}$ pair values equal to $170^{\circ} / 90^{\circ}\left(\mathrm{GO}_{2 / 1} \mathrm{LO}\right)$.

$\theta_{\mathrm{HH}}$, respectively (Figure $5 \mathrm{~b}$ ), this time with a hydrogen pointing toward the instantaneous interface.

For the $\mathrm{GO}_{4 / 1} \mathrm{~L} 1$ layer, the distribution shows a major peak in the region from $50^{\circ}$ to $55^{\circ}$ for $\theta_{\mathrm{DW}}$ and from 140 to $145^{\circ}$ for $\theta_{\mathrm{HH}}$ (a representative structure is shown in Figure 5a). A new minor peak is present around values ranging from $90^{\circ}$ to $95^{\circ}$ for both $\theta_{\mathrm{DW}}$ and $\theta_{\mathrm{HH}}$ (a representative structure is shown in Figure 5c), where both $\mathrm{OH}$ bonds are almost parallel to the instantaneous interface, slightly pointing toward it. The region with values from $145^{\circ}$ to $150^{\circ}$ and from $115^{\circ}$ to $120^{\circ}$ for $\theta_{\mathrm{DW}}$ and $\theta_{\mathrm{HH}}$, respectively, is significantly diminished compared to those for $\mathrm{GO}_{2 / 1}$. Finally, $\mathrm{GO}_{2 / 1}$ presents the same number of $\mathrm{OH}$ bonds pointing away and toward the interface, whereas, in $\mathrm{GO}_{4 / 1}$, most of them are pointing away from the interface or are almost parallel to it.

For the $\mathrm{GO}_{2 / 1}$ L0 layer, the joint distribution is shown in Figure $4 \mathrm{c}$, and only one orientation is seen, with $\theta_{\mathrm{DW}}$ ranging from $170^{\circ}$ to $175^{\circ}$ and a $\theta_{\mathrm{HH}}$ value from $90^{\circ}$ to $95^{\circ}$ (Figure $5 \mathrm{~d}$ ). This configuration has both hydrogens pointing toward the instantaneous surface, which combined with the fact that in L0 water molecules are situated between the graphene sheet and the instantaneous interface, makes these hydrogens effectively pointing away from the graphene sheet.

III.d. vSFG Spectra of the Graphene Oxide-Water Interface. As mentioned in the Introduction, the vSFG spectroscopic technique is highly surface-specific due to its dependence on $\chi^{(2)}$, the second-order nonlinear susceptibility, and is thus zero in a centrosymmetric environment. ${ }^{4,48}$ The experimental intensity (SSP polarization) $I_{\mathrm{ssp}} \propto\left|\chi_{\mathrm{xxz}}^{(2)}\right|^{2}$, where $\chi^{(2)}=\chi^{(2), \mathrm{R}}+\chi^{(2), \mathrm{NR}}$, with the two terms being the resonant $\left(\chi^{(2), \mathrm{R}}\right)$ and nonresonant $\left(\chi^{(2), \mathrm{NR}}\right)$ parts, respectively. At a fixed visible frequency, the nonresonant term is constant. ${ }^{94}$ However, it should be pointed out that there could be a small $\chi^{(3)}$ contribution to the experimental intensity, ${ }^{95,96}$ which was neglected in this work and will be examined in future work.

In Figure 6a, the experimental spectra of the water-GOsapphire interface obtained by SSP polarization are reported. For the unreduced system, there is a major peak in the highfrequency region at $3700 \mathrm{~cm}^{-1}$ and a very broad intensity within the $3200-3500 \mathrm{~cm}^{-1}$ range with a minor peak at 3375 $\mathrm{cm}^{-1}$. In the literature, this peak around $3700 \mathrm{~cm}^{-1}$ is typically attributed to dangling $\mathrm{OH}$ bonds pointing toward the air-
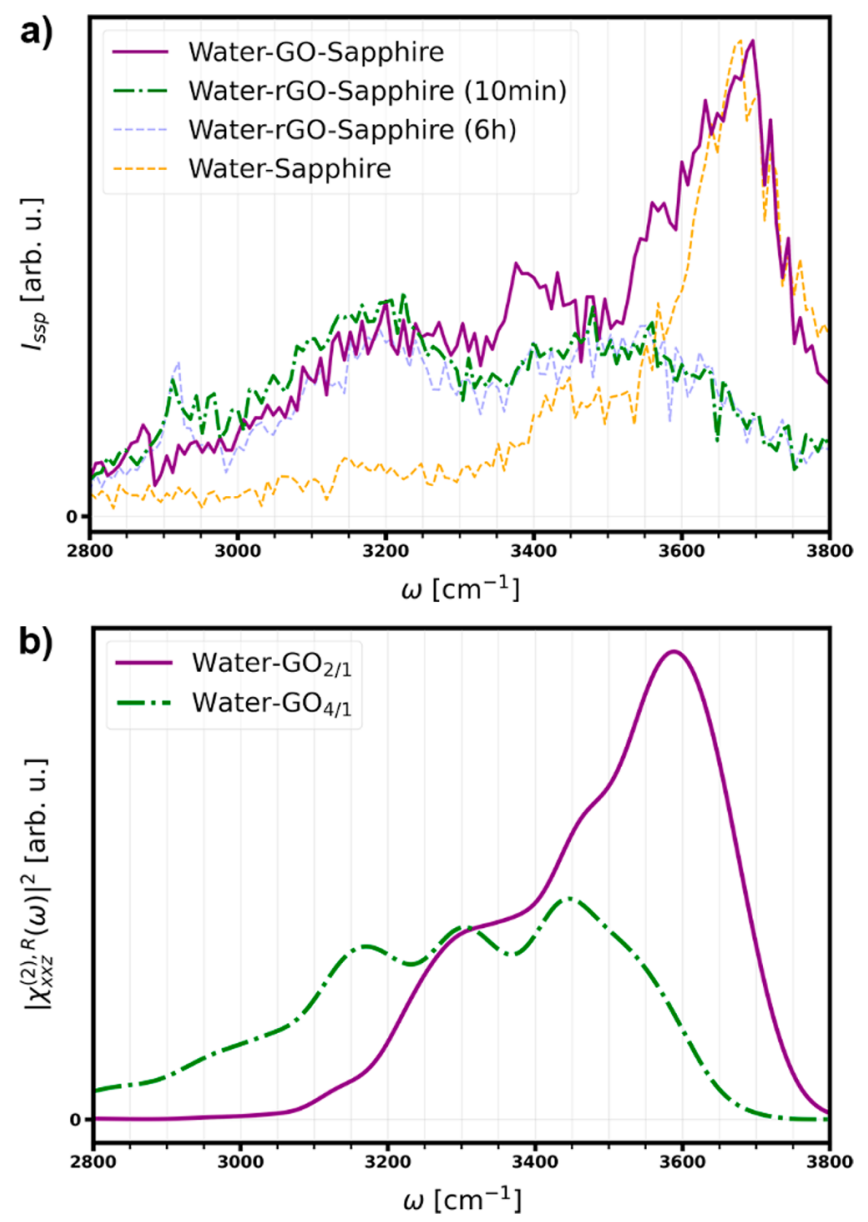

Figure 6. (a) Experimental vSFG spectra of the water-GO interface before and after reduction by SSP polarization. (b) Simulated vSFG spectra of the water- $\mathrm{GO}_{2 / 1}$ and the water- $\mathrm{GO}_{4 / 1}$ interfaces. The simulated spectra were scaled to have the same height of the maximum as the experiment.

water interface ${ }^{50,53,59,97-99}$ or the graphene-water interface, ${ }^{100}$ whereas the range between 3200 and $3500 \mathrm{~cm}^{-1}$ is typically attributed to hydrogen-bonded $\mathrm{OH}$ bonds (from water and hydroxyl groups) whether pointing away from or toward the interface. ${ }^{50,53,59,97-99,101-103}$ After $10 \mathrm{~min}$ of reduction, one can see the disappearance of the $3700 \mathrm{~cm}^{-1}$ peak, a growth of a peak around $3500 \mathrm{~cm}^{-1}$, and a specific peak growing at 2900 $\mathrm{cm}^{-1}$, which can be attributed to methine groups resulting from the reduction of the graphene oxide. ${ }^{104}$ After $6 \mathrm{~h}$ of reduction, the system presents no major difference with the system after $10 \mathrm{~min}$ of reduction. One would expect that the more oxidized graphene oxide would present fewer highfrequency intensities at the interface due to the presence of more oxygen-bearing groups available for hydrogen bonding, resulting in a lower number of weak or free $\mathrm{OH}$ oscillators: this is not the case, thereby underlining the need for molecular simulations of these two systems to obtain insight on the local structure of these interfaces.

A simulated spectrum can be obtained via the surface specific velocity-velocity correlation function formalism proposed by Otho et al. ${ }^{84}$ from molecular dynamics simulations. Here the resonant component of the secondorder susceptibility was calculated. This method ensures a fast convergence, thus preventing the need for very long sampling trajectories. Figure $6 \mathrm{~b}$ shows the simulated $\left|\chi_{\mathrm{xxz}}^{(2), \mathrm{R}}\right|^{2}$ spectra, 

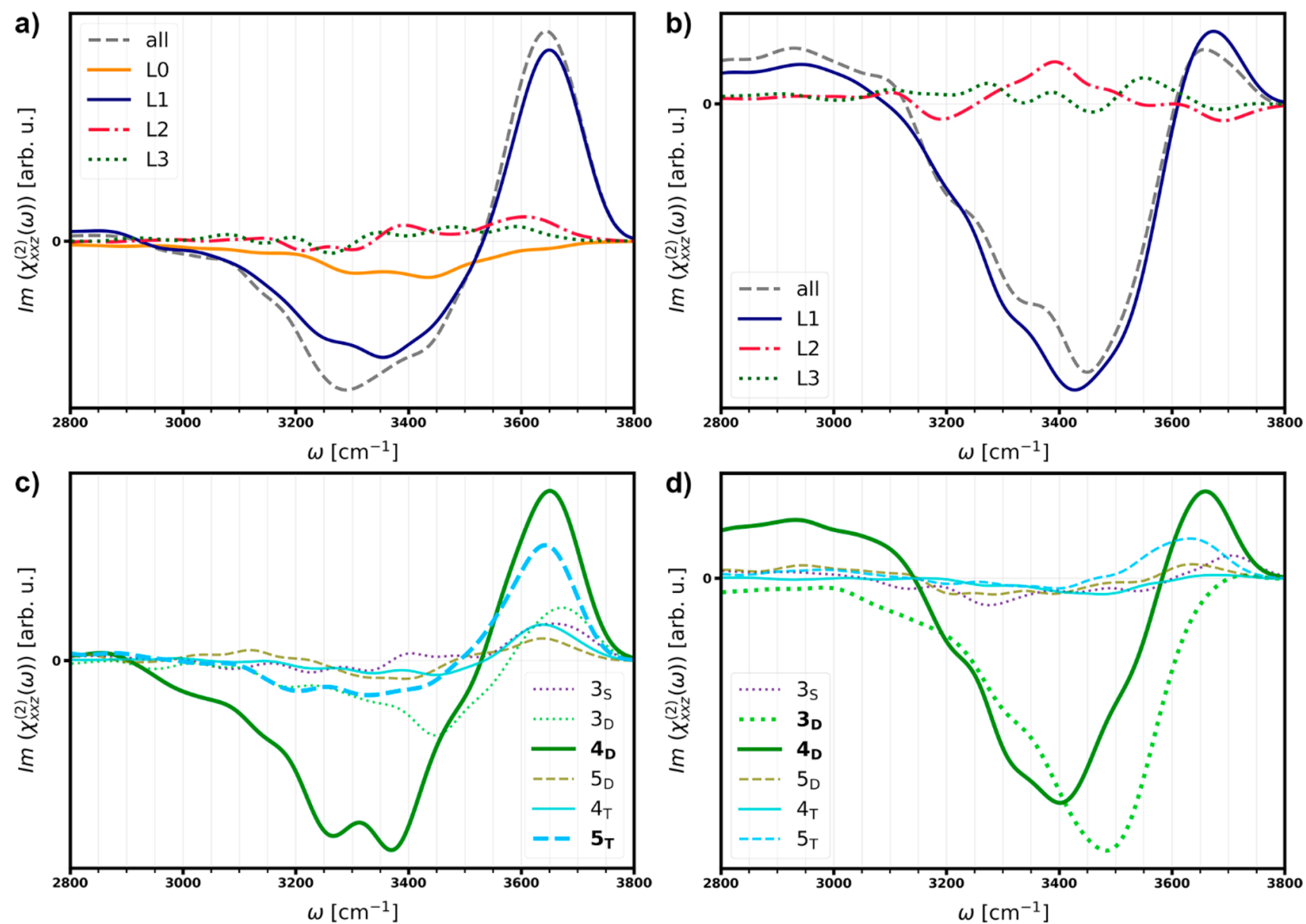

Figure 7. (a) Imaginary part of the simulated vSFG spectra of the water- $\mathrm{GO}_{2 / 1}$ interface by layers. (b) The imaginary part of the simulated vSFG spectra of the water- $\mathrm{GO}_{4 / 1}$ interface by layers. (c) The imaginary part of the simulated vSFG spectra of the water-GO $\mathrm{GO}_{2 / 1}$ interface within the L1 layer by hydrogen-bonding classes. (d) Imaginary part of the simulated vSFG spectra of the water- $\mathrm{GO}_{4 / 1}$ interface within the L1 layer by hydrogenbonding classes.

where only the $\mathrm{OH}$ oscillators from the water (no contribution from GO hydroxyl groups) within $11 \AA$ of the instantaneous interface (to avoid the other interface, namely, the air-water present in the simulation) are taken into account. The simulated spectra with confidence intervals are given in the Supporting Information (Figure S3). The spectrum from the air-water interface can be found in Figure S4 in the Supporting Information and reproduces the experimental spectrum of air-water interface from the literature, further validating the functional used in the AIMD simulations. These simulated systems, compared to the experimental one, possess no sapphire support for the graphene oxide sheet, and any interpenetrated waters between the substrate and GO are absent. ${ }^{100}$ For $\mathrm{GO}_{2 / 1}$, the peaks are slightly red-shifted (100 $\left.\mathrm{cm}^{-1}\right)^{105}$ compared to the experimental results, with a major peak at $3600 \mathrm{~cm}^{-1}$ and a neighboring shoulder at $3300 \mathrm{~cm}^{-1}$. Most of the low-frequency range (lower than $3200 \mathrm{~cm}^{-1}$ ) is absent in the simulated vSFG spectra from $\mathrm{GO}_{2 / 1}$. A possibility is that this region is different due to the noninclusion of any $\mathrm{OH}$ bonds from any hydroxyl groups, which can form hydrogen bonds (and thus appear in this low-frequency range) with other nearby oxygen-bearing groups (alkoxides, epoxides, hydroxyls) as well as water, as seen in previous experimental $^{106}$ and theoretical ${ }^{107,108}$ vSFG studies on mineral-water interfaces. Nevertheless, the dominant features present in the experimental vSFG spectra are well-represented.
For the $\mathrm{GO}_{4 / 1}$ case, the $\left|\chi_{\mathrm{xxz}}^{(2), \mathrm{R}}\right|^{2}$ shows the characteristic loss of the high-frequency dominant peak at $3600 \mathrm{~cm}^{-1}$, consistent with the experimentally reduced $\mathrm{GO}$, and gains three peaks at 3450,3300 , and $3150 \mathrm{~cm}^{-1}$ and a broad region below 3200 $\mathrm{cm}^{-1}$, which means that, for $\mathrm{GO}_{4 / 1}, \mathrm{OH}$ bonds from water also account for this region, suggesting a strong hydrogen-bonding environment not only due to hydroxyl groups. Once again, the simulated spectrum for $\mathrm{GO}_{4 / 1}$ qualitatively reproduces the main features of the experimental vSFG spectrum. To get further insight into the interface, the resonant imaginary component, $\operatorname{Im}_{\chi_{x x z}^{(2)}}$, which is equal to $\operatorname{Im}_{\chi_{x x z}^{(2)}}$, since typically the nonresonant part is real, ${ }^{109}$ was examined. The sign of the imaginary part reflects the direction of the transition dipole (i.e., $\mathrm{OH}$ bond) with respect to the interface: ${ }^{109}$ a positive sign for $\operatorname{Im}_{\chi_{x x z}^{(2)}}$ corresponds to a bond with the $\mathrm{H}$ atom pointing upward (away from the interface), and a negative sign corresponds to an $\mathrm{OH}$ bond with $\mathrm{H}$ pointing downward (toward the interface). Figure $7 \mathrm{a}, \mathrm{b}$ shows the imaginary component $\operatorname{Im}_{\chi_{x x z}^{(2)}}$ for the $\mathrm{GO}_{2 / 1}$ and $\mathrm{GO}_{4 / 1}$ interfaces, and the component for each layer is reported, corresponding to the depth from the interface, and as expected the L1 layer, in both systems, is the major contributing component to $\operatorname{Im}_{\chi_{x x z}^{(2)}}$.

For $\mathrm{GO}_{2 / 1}$, the spectrum of the L0 layer has a negative broad region from $\sim 3100$ to $\sim 3600 \mathrm{~cm}^{-1}$. This is in keeping with the angle distribution in Figure 4c, where all the water molecules 
are pointing away from the graphene-oxide sheet toward the instantaneous surface/interface. This broad negative range is consistent with Figure 3a, where the majority (80\%) of water molecules are double (or more) donors, presenting very few "free" or weakly hydrogen-bonded $\mathrm{OH}$ bonds and are oriented away from the interface.

When looking at the $\mathrm{L} 1$ layer, $\mathrm{GO}_{2 / 1}$ shows a negative region from $\sim 3200$ to $\sim 3500 \mathrm{~cm}^{-1}$ and a sharper positive region at $\sim 3600 \mathrm{~cm}^{-1}$, which are in keeping with the angle distribution in Figure $4 a$, where the water presents both orientations: one with the water oriented with the $\mathrm{H}$ of the $\mathrm{OH}$ bond pointing away from the instantaneous interface (Figure 5a) thereby contributing to the negative region in the imaginary spectrum and another one with, this time, an $\mathrm{OH}$ bond with the $\mathrm{H}$ pointing toward (Figure $5 \mathrm{~b}$ ) the GO sheet and the instantaneous interface (contributing to the positive peak at higher frequencies).

For the L1 layer of $\mathrm{GO}_{4 / 1}$ (Figure $4 \mathrm{~b}$ ), $\operatorname{Im}_{\chi_{x z}^{(2)}}$ presents a major broad negative peak centered at $\sim 3400 \mathrm{~cm}^{-1}$, a small positive peak at $\sim 3700 \mathrm{~cm}^{-1}$, and a small positive component in the low-frequency region (less than $3000 \mathrm{~cm}^{-1}$ ). This is consistent with the angle distribution with most waters having an $\mathrm{OH}$ pointing away from the surface (Figure $4 \mathrm{~b}$ and Figure 5a).

For further insight, $\operatorname{Im}_{\chi_{x x z}}{ }^{(2)}$ is reported in Figure $7 \mathrm{c}, \mathrm{d}$ for each major water hydrogen-bond class in the $\mathrm{L} 1$ layer for $\mathrm{GO}_{2 / 1}$ and $\mathrm{GO}_{4 / 1}$, respectively. The $\mathbf{4}_{\mathrm{D}}$ class of water, which is the major class for both systems, gives rise to markedly different spectra in the two cases. Additionally, the other two hydrogen-bonding classes that contribute to the spectrum of $\mathrm{GO}_{2 / 1}$ are $\mathbf{3}_{\mathrm{D}}$ and $\mathbf{5}_{\mathrm{T}}$, whereas for the $\mathrm{GO}_{4 / 1}$ case the only other major contribution apart from $4_{D}$ waters is from the $3_{D}$ waters.

The $\theta_{\mathrm{DW}} / \theta_{\mathrm{HH}}$ joint distribution for the $4_{\mathrm{D}}$ waters in the $\mathrm{L} 1$ layer for the two GO interfaces (Figure S5a,b) clearly shows a broad distribution for the $\mathrm{GO}_{2 / 1}$ case, while for the $\mathrm{GO}_{4 / 1}$ case the waters are predominantly such that one $\mathrm{OH}$ bond points away from the interface with the other parallel to the interface (Figure 5a) with a minor peak with the waters almost parallel to the interface but pointing slightly toward the interface. This is in keeping with the $\operatorname{Im}_{\chi_{x \mathrm{xz}}}{ }^{(2)}$ spectra for the $4_{D}$ waters in the L1 layer, which has large positive and negative contributions for the $\mathrm{GO}_{2 / 1}$ case, whereas the positive contribution is considerably dampened in the $\mathrm{GO}_{4 / 1}$ case. The $3_{\mathrm{D}}$ waters also show a broad distribution (Figure $\mathrm{S} 5 \mathrm{c}, \mathrm{d}$ ) for the $\mathrm{GO}_{2 / 1}$ case, whereas for the $\mathrm{GO}_{4 / 1}$ case the waters are oriented with one $\mathrm{OH}$ pointing away from the interface and the other parallel to the interface or a minor peak with waters almost parallel but pointing slightly away from the interface. This again reflects the features of the $\operatorname{Im}_{\chi_{x \mathrm{x}}}{ }^{(2)}$ spectra for the two interfaces with a positive and negative region for the $\mathrm{GO}_{2 / 1}$ case but only a negative region for the $\mathrm{GO}_{4 / 1}$ case. The $\mathbf{5}_{\mathrm{T}}$ case (Figure S5e,f) has waters for both interfaces essentially oriented with one $\mathrm{OH}$ pointing toward the interface and the other parallel to the interface corresponding to an essentially strong positive feature in the spectrum.

Closer examination of the hydrogen bonds of the $\mathbf{4}_{\mathrm{D}}$ waters that are within the first maxima of the $\mathrm{L} 1$ region (distance from the instantaneous surface is less than or equal to the first peak, $1.75 \AA$ for $\mathrm{GO}_{2 / 1}, 1.25 \AA$ for $\mathrm{GO}_{4 / 1}$ ) show some interesting features (see the Supporting Information for the definition of the $\mathrm{r}-\alpha$ pair). For the $\mathrm{OH}_{\mathrm{D}}$ donor vectors pointing toward the interface $\left(\theta_{\mathrm{OH}} \geq 110^{\circ}\right)$, the joint distribution of the hydrogen- bond distance $(r)$ and hydrogen-bond angles $(\alpha)$ (Figure 8a) show significant deviation from the bulk water case (Figure S6)

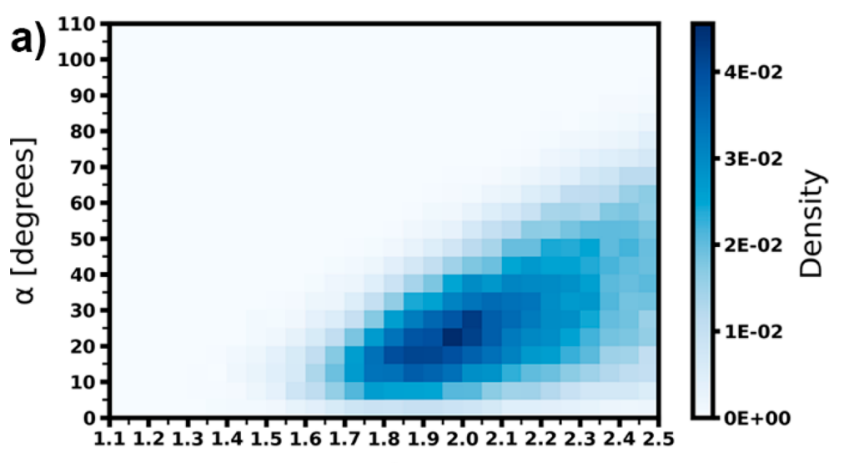

$r[\AA]$

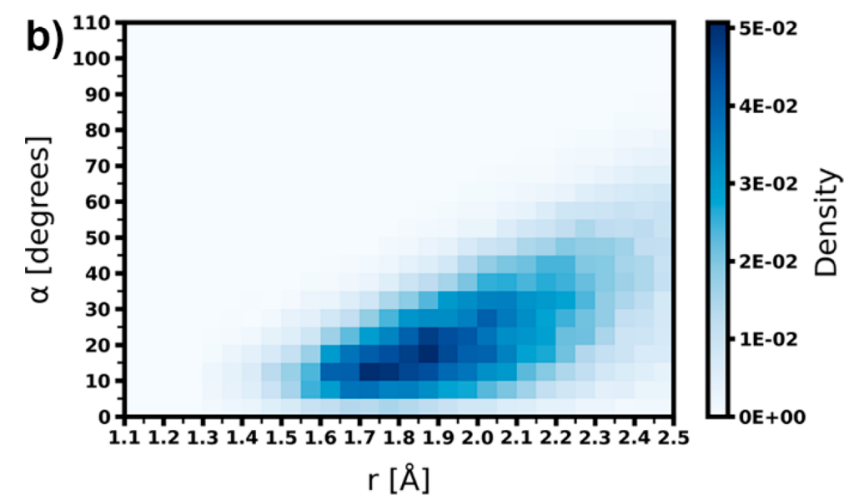

Figure 8. Two-dimensional histograms of the joint distribution of the $r$ - $\alpha$ pair for hydrogen bonds with donating L1 $4_{D}$ water (and where their distance to the instantaneous surface is less or equal to the first density peak, $1.75 \AA$ for $\mathrm{GO}_{2 / 1}, 1.25 \AA$ for $\left.\mathrm{GO}_{4 / 1}\right)$. Only hydrogen bonds with the $\mathrm{OH}_{\mathrm{D}}$ donor vector pointing toward the interface $\left(\theta_{\mathrm{OH}}\right.$ $\geq 110^{\circ}$ ) are considered. (a) $\mathrm{GO}_{2 / 1}$. (b) $\mathrm{GO}_{4 / 1}$. All distributions were normalized to have a unit area.

for $\mathrm{GO}_{2 / 1}$ with larger angles and longer distances, suggesting that these hydrogen bonds are much weaker than the case of bulk water and hence are much less red-shifted. This effect is less pronounced for the $\mathrm{GO}_{4 / 1}$ case (Figure $8 \mathrm{~b}$ ), and coupled with the fact that these orientations are far fewer in the $\mathrm{GO}_{4 / 1}$ case, the positive feature at high frequencies is considerably dampened.

From this decomposition analysis, one can see that, in addition to the differences in the distribution of the hydrogenbonding classes of water, the interfacial waters at the $\mathrm{GO}_{2 / 1^{-}}$ and $\mathrm{GO}_{4 / 1}$-water interfaces are oriented differently depending on the oxidation level of the graphene-oxide sheet resulting in very different vSFG spectra. The combined analysis of the orientation and hydrogen-bonding environment gives substantial insight into the type of interaction with water and the graphene-oxide surface. The $5_{\mathrm{T}}$ class, increasing only by $2.4 \%$ between $\mathrm{GO}_{4 / 1}$ and $\mathrm{GO}_{2 / 1}$ and accounting for less than $10 \%$, becomes the second most-dominant feature of the spectra for $\mathrm{GO}_{2 / 1}$, and the $3_{\mathrm{D}}$ class decreasing from $24.9 \%$ to $16.3 \%$ in $\mathrm{GO}_{2 / 1}$, still being the second most-populous class, is not a dominant feature any more in the vSFG spectra. The $\mathbf{4}_{\mathrm{D}}$ class, varying from $44.5 \%$ to $42.0 \%$, drastically changes its contribution to the vSFG spectra between $\mathrm{GO}_{4 / 1}$ and $\mathrm{GO}_{2 / 1}$.

III.e. Reactivity of the GO-Water Interface. The AIMD simulations reveal several spontaneous epoxide (Figure 9a) opening events forming a charged pair of an alkoxide ion and a 
a)

b)

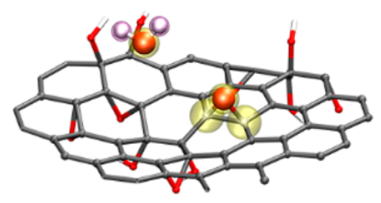

c)
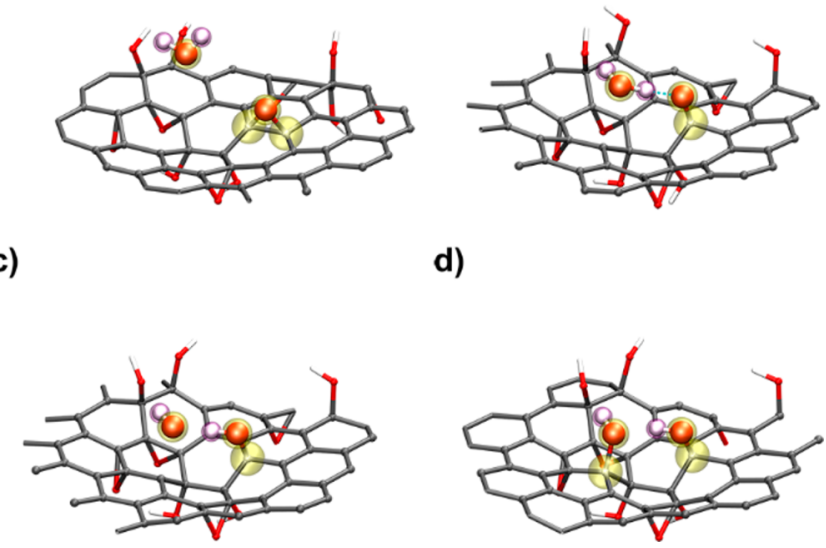

d)

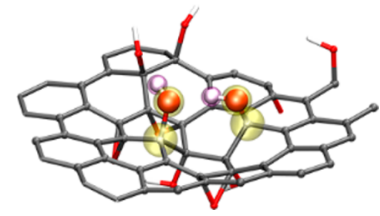

Figure 9. Reactive event chain in the $\mathrm{GO}_{4 / 1}$. (a) Epoxide and water. (b) Epoxide opening to form an alkoxy and a carbocation. (c) Water hydrogen abstraction by the alkoxy to form a hydroxy group. (d) Addition of the hydroxide (previously formed from the water) on the carbocation to form a new hydroxy group. Reactive species are highlighted and are represented with ball and sticks. The other part of the GO surface is represented with sticks. Only a portion of the GO sheet is shown with just the reactive water (the remaining waters and GO sheet are not shown for the sake of clarity). Carbons, oxygens, and hydrogens are represented in gray, red, and white, respectively.

delocalized carbocation on the GO sheet (Figure 9b), highlighting the reactive nature of the GO surface. These alkoxides are further stabilized by hydrogen bonds from both the hydroxyl groups of GO and water, and Figure S7 in the Supporting Information shows that the hydrogen bonds formed by water with the alkoxide are very strong (unlike the other oxygenated groups) leading to red-shifts in the $\mathrm{OH}$ spectra. The alkoxide ions can abstract a proton from a neighboring hydroxyl group forming a hydroxyl group and a new alkoxide, resulting in the shuttling of the alkoxide along the sheet. Furthermore, reactive events in which the alkoxide extracts a proton from water forming a hydroxyl group are also seen (Figure 9c).

This could also be the origin of the positive red-shifted region in the water spectrum of $\mathrm{GO}_{4 / 1}$, since the $\mathrm{OH}$ group of the reactive water has a partial hydroxyl group character. Interestingly, the decomposition of the imaginary spectrum of water $\mathrm{O}-\mathrm{Hs}$ in the L1 layer that participate or do not participate in hydrogen bonds with the GO surface shows that the $\mathrm{O}-\mathrm{H}$ waters that are involved in hydrogen bonds (specifically donor hydrogen bonds) give rise to this redshifted feature (see Figures S8 and S9) in $\mathrm{GO}_{4 / 1}$. The hydroxide ion that is formed can then attack the carbocation forming another hydroxyl group (Figure 9d). These ringopening events that create alkoxide species result in carbocations that are stabilized by the graphene-rich regions that are present in $\mathrm{GO}_{4 / 1}$. Hence it is unsurprising that, for $\mathrm{GO}_{4 / 1}$, the ratio of alkoxide to oxygen-bearing groups is 0.066 , and for $\mathrm{GO}_{2 / 1}$, it is only 0.030 , and hence reactive events are more likely in the $\mathrm{GO}_{4 / 1}$ case due to the two separate domains. Future studies will further examine these reactive events.

\section{CONCLUSION}

This paper demonstrates that the orientation and the hydrogen-bonding class of water molecules plays a major

role in the vSFG spectra and sheds light on the interactions specific to this interface. The ab initio MD simulations are in good agreement with the experiments, highlighting the fact that the DFT method used here is adequate for this system and details how the water molecules are adapting to the different levels of oxidation of the graphene-oxide sheet. It also provides insight into the interpretation of experimental spectra at a molecular level. A highlight of this work is the fact that this surface is reactive, with epoxide opening and alkoxide shuttling as well as proton abstraction events from interfacial waters, which will be the focus of future studies.

\section{ASSOCIATED CONTENT}

\section{Supporting Information}

The Supporting Information is available free of charge at https://pubs.acs.org/doi/10.1021/acs.jpcb.0c05282.

Additional details and results for the hydrogen bond definition, details of the switching function used in the ssVVCF scheme, simulated air-water vSFG spectrum, joint angle distribution, and joint hydrogen definition pair (PDF)

\section{AUTHOR INFORMATION}

\section{Corresponding Authors}

Revati Kumar - Department of Chemistry, Louisiana State University, Baton Rouge, Louisiana 70803, United States; ๑ orcid.org/0000-0002-3272-8720; Email: revatik@lsu.edu

Aashish Tuladhar - Physical Sciences Division, Physical and Computational Sciences Directorate, Pacific Northwest National Laboratory, Richland, Washington 99352, United States; ๑ orcid.org/0000-0003-2449-4984;

Email: aashish.tuladhar@pnnl.gov

\section{Authors}

Rolf David - Department of Chemistry, Louisiana State University, Baton Rouge, Louisiana 70803, United States; ๑ orcid.org/0000-0001-5338-6267

Le Zhang - Cain Department of Chemical Engineering, Louisiana State University, Baton Rouge, Louisiana 70803, United States

Christopher Arges - Cain Department of Chemical Engineering, Louisiana State University, Baton Rouge, Louisiana 70803, United States; ๑ orcid.org/0000-0003-1703-8323

Complete contact information is available at: https://pubs.acs.org/10.1021/acs.jpcb.0c05282

\section{Author Contributions}

All authors have given approval to the final version of the manuscript.

\section{Notes}

The authors declare no competing financial interest.

\section{ACKNOWLEDGMENTS}

R.D. and R.K. acknowledge support from the National Science Foundation, Grant No. CHE-1845795, as well as LSU-HPC and LONI for computer time. A.T. acknowledges the support provided by the U.S. Department of Energy (DOE), Office of Science, Office of Basic Energy Sciences, Materials Sciences and Engineering Division and Chemical Sciences, Geosciences, and Biosciences Division at The Pacific Northwest National Laboratory (PNNL), operated by Battelle for the U.S. DOE under Contract No. DE-AC05-76RL01830. vSFG experiments 
were performed at the Environmental Molecular Sciences Laboratory, a DOE office of Science User Facility sponsored by the Office of Biological and Environmental Research that is located at PNNL.

\section{REFERENCES}

(1) Zhao, G.; Jiang, L.; He, Y.; Li, J.; Dong, H.; Wang, X.; Hu, W. Sulfonated Graphene for Persistent Aromatic Pollutant Management. Adv. Mater. 2011, 23 (34), 3959-3963.

(2) Chen, D.; Feng, H.; Li, J. Graphene Oxide: Preparation, Functionalization, and Electrochemical Applications. Chem. Rev. 2012, 112 (11), 6027-6053.

(3) Su, C.; Acik, M.; Takai, K.; Lu, J.; Hao, S.; Zheng, Y.; Wu, P.; Bao, Q.; Enoki, T.; Chabal, Y. J.; Ping Loh, K. Probing the Catalytic Activity of Porous Graphene Oxide and the Origin of This Behaviour. Nat. Commun. 2012, 3 (1), 1298.

(4) Gao, Y.; Li, Y.; Zhang, L.; Huang, H.; Hu, J.; Shah, S. M.; Su, X. Adsorption and Removal of Tetracycline Antibiotics from Aqueous Solution by Graphene Oxide. J. Colloid Interface Sci. 2012, 368 (1), 540-546.

(5) Madadrang, C. J.; Kim, H. Y.; Gao, G.; Wang, N.; Zhu, J.; Feng, H.; Gorring, M.; Kasner, M. L.; Hou, S. Adsorption Behavior of EDTA-Graphene Oxide for Pb (II) Removal. ACS Appl. Mater. Interfaces 2012, 4 (3), 1186-1193.

(6) Dhakshinamoorthy, A.; Alvaro, M.; Concepcion, P.; Garcia, H.; Fornés, V. Graphene Oxide as an Acid Catalyst for the Room Temperature Ring Opening of Epoxides. Chem. Commun. 2012, 48 (44), 5443-5445.

(7) Liu, F.; Chung, S.; Oh, G.; Seo, T. S. Three-Dimensional Graphene Oxide Nanostructure for Fast and Efficient Water-Soluble Dye Removal. ACS Appl. Mater. Interfaces 2012, 4 (2), 922-927.

(8) Huang, H.; Song, Z.; Wei, N.; Shi, L.; Mao, Y.; Ying, Y.; Sun, L.; $\mathrm{Xu}, \mathrm{Z}$.; Peng, X. Ultrafast Viscous Water Flow through NanostrandChannelled Graphene Oxide Membranes. Nat. Commun. 2013, 4 (1), 2979.

(9) You, S.; Yu, J.; Sundqvist, B.; Belyaeva, L. A.; Avramenko, N. V.; Korobov, M. V.; Talyzin, A. V. Selective Intercalation of Graphite Oxide by Methanol in Water/Methanol Mixtures. J. Phys. Chem. C 2013, 117 (4), 1963-1968.

(10) Romanchuk, A. Y.; Slesarev, A. S.; Kalmykov, S. N.; Kosynkin, D. V.; Tour, J. M. Graphene Oxide for Effective Radionuclide Removal. Phys. Chem. Chem. Phys. 2013, 15 (7), 2321.

(11) DeYoung, A. D.; Park, S.-W.; Dhumal, N. R.; Shim, Y.; Jung, Y.; Kim, H. J. Graphene Oxide Supercapacitors: A Computer Simulation Study. J. Phys. Chem. C 2014, 118 (32), 18472-18480.

(12) Kim, D.; Kim, D. W.; Lim, H.-K.; Jeon, J.; Kim, H.; Jung, H.-T.; Lee, $\mathrm{H}$. Intercalation of Gas Molecules in Graphene Oxide Interlayer: The Role of Water. J. Phys. Chem. C 2014, 118 (20), 11142-11148.

(13) Ban, S.; Jing, X.; Zhou, H.; Zhang, L.; Zhang, J. Experimental and Modeling Study on Charge Storage/Transfer Mechanism of Graphene-Based Supercapacitors. J. Power Sources 2014, 268, 604609.

(14) Martins, M. V. A.; Pereira, A. R.; Luz, R. A. S.; Iost, R. M.; Crespilho, F. N. Evidence of Short-Range Electron Transfer of a Redox Enzyme on Graphene Oxide Electrodes. Phys. Chem. Chem. Phys. 2014, 16 (33), 17426-17436.

(15) Smith, Z. P.; Freeman, B. D. Graphene Oxide: A New Platform for High-Performance Gas- and Liquid-Separation. Angew. Chem., Int. Ed. 2014, 53 (39), 10286-10288.

(16) Gao, W.; Wu, G.; Janicke, M. T.; Cullen, D. A.; Mukundan, R.; Baldwin, J. K.; Brosha, E. L.; Galande, C.; Ajayan, P. M.; More, K. L.; Dattelbaum, A. M.; Zelenay, P. Ozonated Graphene Oxide Film as a Proton-Exchange Membrane. Angew. Chem., Int. Ed. 2014, 53 (14), 3588-3593.

(17) Ray, S. Applications of Graphene and Graphene-Oxide Based Nanomaterials; Elsevier, 2015.
(18) Lee, O.-S.; Carignano, M. A. Exfoliation of ElectrolyteIntercalated Graphene: Molecular Dynamics Simulation Study. J. Phys. Chem. C 2015, 119 (33), 19415-19422.

(19) Wang, J.; Chen, B. Adsorption and Coadsorption of Organic Pollutants and a Heavy Metal by Graphene Oxide and Reduced Graphene Materials. Chem. Eng. J. 2015, 281, 379-388.

(20) Guo, H.; Jiao, T.; Zhang, Q.; Guo, W.; Peng, Q.; Yan, X. Preparation of Graphene Oxide-Based Hydrogels as Efficient Dye Adsorbents for Wastewater Treatment. Nanoscale Res. Lett. 2015, 10 (1), 272.

(21) Xu, Q.; Xu, H.; Chen, J.; Lv, Y.; Dong, C.; Sreeprasad, T. S. Graphene and Graphene Oxide: Advanced Membranes for Gas Separation and Water Purification. Inorg. Chem. Front. 2015, 2 (5), $417-424$.

(22) Xu, K.; Ji, X.; Chen, C.; Wan, H.; Miao, L.; Jiang, J. Electrochemical Double Layer near Polar Reduced Graphene Oxide Electrode: Insights from Molecular Dynamic Study. Electrochim. Acta 2015, 166, 142-149.

(23) Hegab, H. M.; Zou, L. Graphene Oxide-Assisted Membranes: Fabrication and Potential Applications in Desalination and Water Purification. J. Membr. Sci. 2015, 484, 95-106.

(24) Li, W.; Zheng, X.; Dong, Z.; Li, C.; Wang, W.; Yan, Y.; Zhang, J. Molecular Dynamics Simulations of CO 2 /N 2 Separation through Two-Dimensional Graphene Oxide Membranes. J. Phys. Chem. C 2016, 120 (45), 26061-26066.

(25) Park, S.-W.; DeYoung, A. D.; Dhumal, N. R.; Shim, Y.; Kim, H. J.; Jung, Y. Computer Simulation Study of Graphene Oxide Supercapacitors: Charge Screening Mechanism. J. Phys. Chem. Lett. 2016, 7 (7), 1180-1186.

(26) Abraham, J.; Vasu, K. S.; Williams, C. D.; Gopinadhan, K.; Su, Y.; Cherian, C. T.; Dix, J.; Prestat, E.; Haigh, S. J.; Grigorieva, I. V.; Carbone, P.; Geim, A. K.; Nair, R. R. Tunable Sieving of Ions Using Graphene Oxide Membranes. Nat. Nanotechnol. 2017, 12 (6), 546550.

(27) Pandey, R. P.; Shukla, G.; Manohar, M.; Shahi, V. K. Graphene Oxide Based Nanohybrid Proton Exchange Membranes for Fuel Cell Applications: An Overview. Adv. Colloid Interface Sci. 2017, 240, 1530 .

(28) Sun, Y.; Tang, J.; Zhang, K.; Yuan, J.; Li, J.; Zhu, D.-M. M.; Ozawa, K.; Qin, L.-C. C. Comparison of Reduction Products from Graphite Oxide and Graphene Oxide for Anode Applications in Lithium-Ion Batteries and Sodium-Ion Batteries. Nanoscale 2017, 9 (7), 2585-2595.

(29) Thakur, K.; Kandasubramanian, B. Graphene and Graphene Oxide-Based Composites for Removal of Organic Pollutants: A Review. J. Chem. Eng. Data 2019, 64 (3), 833-867.

(30) Chen, Y.; Luo, Z.; Lu, X. Construction of Novel EnzymeGraphene Oxide Catalytic Interface with Improved Enzymatic Performance and Its Assembly Mechanism. ACS Appl. Mater. Interfaces 2019, 11 (12), 11349-11359.

(31) Witomska, S.; Liu, Z.; Czepa, W.; Aliprandi, A.; Pakulski, D.; Pawluć, P.; Ciesielski, A.; Samorì, P. Graphene Oxide Hybrid with Sulfur-Nitrogen Polymer for High-Performance Pseudocapacitors. J. Am. Chem. Soc. 2019, 141 (1), 482-487.

(32) Dong, Y.; Cheng, Y.; Xu, G.; Cheng, H.; Huang, K.; Duan, J.; Mo, D.; Zeng, J.; Bai, J.; Sun, Y.; Liu, J.; Yao, H. Selectively Enhanced Ion Transport in Graphene Oxide Membrane/PET Conical Nanopore System. ACS Appl. Mater. Interfaces 2019, 11 (16), 1496014969.

(33) Yang, H.; Yeow, B. S.; Chang, T.-H.; Li, K.; Fu, F.; Ren, H.; Chen, P.-Y. Graphene Oxide-Enabled Synthesis of Metal Oxide Origamis for Soft Robotics. ACS Nano 2019, 13 (5), 5410-5420.

(34) Qiu, R.; Yuan, S.; Xiao, J.; Chen, X. D.; Selomulya, C.; Zhang, X.; Woo, M. W. Effects of Edge Functional Groups on Water Transport in Graphene Oxide Membranes. ACS Appl. Mater. Interfaces 2019, 11 (8), 8483-8491.

(35) Yoon, H. W.; Lee, T. H.; Doherty, C. M.; Choi, T. H.; Roh, J. S.; Kim, H. W.; Cho, Y. H.; Do, S.; Freeman, B. D.; Park, H. B. Origin 
of CO 2 -Philic Sorption by Graphene Oxide Layered Nanosheets and Their Derivatives. J. Phys. Chem. Lett. 2020, 11 (6), 2356-2362.

(36) Garg, B.; Bisht, T.; Ling, Y.-C. Graphene-Based Nanomaterials as Heterogeneous Acid Catalysts: A Comprehensive Perspective. Molecules 2014, 19 (9), 14582-14614.

(37) He, H.; Klinowski, J.; Forster, M.; Lerf, A. A New Structural Model for Graphite Oxide. Chem. Phys. Lett. 1998, 287 (1-2), 5356.

(38) Lerf, A.; He, H.; Forster, M.; Klinowski, J. Structure of Graphite Oxide Revisited. J. Phys. Chem. B 1998, 102 (23), 4477-4482.

(39) Lerf, A.; He, H.; Riedl, T.; Forster, M.; Klinowski, J. 13C and 1H MAS NMR Studies of Graphite Oxide and Its Chemically Modified Derivatives. Solid State Ionics 1997, 101-103, 857-862.

(40) Motevalli, B.; Parker, A. J.; Sun, B.; Barnard, A. S. The Representative Structure of Graphene Oxide Nanoflakes from Machine Learning. Nano Futures 2019, 3 (4), 045001.

(41) Sinclair, R. C.; Coveney, P. V. Modeling Nanostructure in Graphene Oxide: Inhomogeneity and the Percolation Threshold. J. Chem. Inf. Model. 2019, 59 (6), 2741-2745.

(42) Zaera, F. Probing Liquid/Solid Interfaces at the Molecular Level. Chem. Rev. 2012, 112 (5), 2920-2986.

(43) Flynn Bolte, K. T.; Balaraman, R. P.; Jiao, K.; Tustison, M.; Kirkwood, K. S.; Zhou, C.; Kohli, P. Probing Liquid-Solid and Vapor-Liquid-Solid Interfaces of Hierarchical Surfaces Using HighResolution Microscopy. Langmuir 2018, 34 (12), 3720-3730.

(44) Woodruff, D. P.; Delchar, T. A. Modern Techniques of Surface Science; Cambridge University Press: Cambridge, UK, 1994.

(45) Turner, N. H.; Dunlap, B. I.; Colton, R. J. Surface Analysis: XRay Photoelectron Spectroscopy, Auger Electron Spectroscopy and Secondary Ion Mass Spectrometry. Anal. Chem. 1984, 56 (5), 373416.

(46) Belsey, N. A.; Shard, A. G.; Minelli, C. Surface Chemistry. In Nanomaterial Characterization; John Wiley \& Sons, Inc: Hoboken, NJ, 2016; pp 153-178.

(47) Hunt, J. H.; Guyot-Sionnest, P.; Shen, Y. R. Observation of C$\mathrm{H}$ Stretch Vibrations of Monolayers of Molecules Optical SumFrequency Generation. Chem. Phys. Lett. 1987, 133 (3), 189-192.

(48) Guyot-Sionnest, P.; Hunt, J. H.; Shen, Y. R. Sum-Frequency Vibrational Spectroscopy of a Langmuir Film: Study of Molecular Orientation of a Two-Dimensional System. Phys. Rev. Lett. 1987, 59 (14), 1597-1600.

(49) Stiopkin, I. V.; Jayathilake, H. D.; Bordenyuk, A. N.; Benderskii, A. V. Heterodyne-Detected Vibrational Sum Frequency Generation Spectroscopy. J. Am. Chem. Soc. 2008, 130 (7), 2271-2275.

(50) Nihonyanagi, S.; Yamaguchi, S.; Tahara, T. Direct Evidence for Orientational Flip-Flop of Water Molecules at Charged Interfaces: A Heterodyne-Detected Vibrational Sum Frequency Generation Study. J. Chem. Phys. 2009, 130 (20), 204704.

(51) Nagata, Y.; Hsieh, C.-S.; Hasegawa, T.; Voll, J.; Backus, E. H. G.; Bonn, M. Water Bending Mode at the Water-Vapor Interface Probed by Sum-Frequency Generation Spectroscopy: A Combined Molecular Dynamics Simulation and Experimental Study. J. Phys. Chem. Lett. 2013, 4 (11), 1872-1877.

(52) Perakis, F.; De Marco, L.; Shalit, A.; Tang, F.; Kann, Z. R.; Kühne, T. D.; Torre, R.; Bonn, M.; Nagata, Y. Vibrational Spectroscopy and Dynamics of Water. Chem. Rev. 2016, 116 (13), $7590-7607$.

(53) Balzerowski, P.; Meister, K.; Versluis, J.; Bakker, H. J. Heterodyne-Detected Sum Frequency Generation Spectroscopy of Polyacrylic Acid at the Air/Water-Interface. Phys. Chem. Chem. Phys. 2016, 18 (4), 2481-2487.

(54) Singla, S.; Anim-Danso, E.; Islam, A. E.; Ngo, Y.; Kim, S. S.; Naik, R. R.; Dhinojwala, A. Insight on Structure of Water and Ice Next to Graphene Using Surface-Sensitive Spectroscopy. ACS Nano 2017, 11 (5), 4899-4906.

(55) Wilson, M. C.; Singla, S.; Stefin, A. J.; Kaur, S.; Brown, J. V.; Dhinojwala, A. Characterization of Acid-Base Interactions Using Interface-Sensitive Sum Frequency Generation Spectroscopy. J. Phys. Chem. C 2019, 123 (30), 18495-18501.
(56) Link, K. A.; Spurzem, G. N.; Tuladhar, A.; Chase, Z.; Wang, Z.; Wang, H.; Walker, R. A. Organic Enrichment at Aqueous Interfaces: Cooperative Adsorption of Glucuronic Acid to DPPC Monolayers Studied with Vibrational Sum Frequency Generation. J. Phys. Chem. A 2019, 123 (26), 5621-5632.

(57) Tuladhar, A.; Chase, Z. A.; Baer, M. D.; Legg, B. A.; Tao, J.; Zhang, S.; Winkelman, A. D.; Wang, Z.; Mundy, C. J.; De Yoreo, J. J.; Wang, H. Direct Observation of the Orientational Anisotropy of Buried Hydroxyl Groups inside Muscovite Mica. J. Am. Chem. Soc. 2019, 141 (5), 2135-2142.

(58) Morita, A.; Ishiyama, T. Recent Progress in Theoretical Analysis of Vibrational Sum Frequency Generation Spectroscopy. Phys. Chem. Chem. Phys. 2008, 10 (38), 5801.

(59) Auer, B. M.; Skinner, J. L. Vibrational Sum-Frequency Spectroscopy of the Liquid/Vapor Interface for Dilute HOD in D2O. J. Chem. Phys. 2008, 129 (21), 214705.

(60) Sulpizi, M.; Salanne, M.; Sprik, M.; Gaigeot, M.-P. Vibrational Sum Frequency Generation Spectroscopy of the Water Liquid-Vapor Interface from Density Functional Theory-Based Molecular Dynamics Simulations. J. Phys. Chem. Lett. 2013, 4 (1), 83-87.

(61) Kishinaka, S.; Morita, A.; Ishiyama, T. Molecular Structure and Vibrational Spectra at Water/Poly(2-Methoxyethylacrylate) and Water/Poly(Methyl Methacrylate) Interfaces: A Molecular Dynamics Simulation Study. J. Chem. Phys. 2019, 150 (4), 044707.

(62) Tuladhar, A.; Dewan, S.; Pezzotti, S.; Brigiano, F. S.; Creazzo, F.; Gaigeot, M.-P.; Borguet, E. Ions Tune Interfacial Water Structure and Modulate Hydrophobic Interactions at Silica Surfaces. J. Am. Chem. Soc. 2020, 142, 6991.

(63) Martínez, L.; Andrade, R.; Birgin, E. G.; Martínez, J. M. PACKMOL: A Package for Building Initial Configurations for Molecular Dynamics Simulations. J. Comput. Chem. 2009, 30 (13), 2157-2164.

(64) Jewett, A. I.; Zhuang, Z.; Shea, J.-E. Moltemplate a CoarseGrained Model Assembly Tool. Biophys. J. 2013, 104 (2), 169a.

(65) Jorgensen, W. L.; Maxwell, D. S.; Tirado-Rives, J. Development and Testing of the OPLS All-Atom Force Field on Conformational Energetics and Properties of Organic Liquids. J. Am. Chem. Soc. 1996, 118 (45), 11225-11236.

(66) Berendsen, H. J. C.; Grigera, J. R.; Straatsma, T. P. The Missing Term in Effective Pair Potentials. J. Phys. Chem. 1987, 91 (24), 62696271.

(67) Hockney, R. .; Eastwood, J. . Computer Simulation Using Particles; Taylor \& Francis, 1988.

(68) Ryckaert, J.-P.; Ciccotti, G.; Berendsen, H. J. Numerical Integration of the Cartesian Equations of Motion of a System with Constraints: Molecular Dynamics of n-Alkanes. J. Comput. Phys. 1977, 23 (3), 327-341.

(69) Nosé, S. A Molecular Dynamics Method for Simulations in the Canonical Ensemble. Mol. Phys. 1984, 52 (2), 255-268.

(70) Hoover, W. G. Canonical Dynamics: Equilibrium Phase-Space Distributions. Phys. Rev. A: At., Mol., Opt. Phys. 1985, 31 (3), 16951697.

(71) VandeVondele, J.; Krack, M.; Mohamed, F.; Parrinello, M.; Chassaing, T.; Hutter, J. Quickstep: Fast and Accurate Density Functional Calculations Using a Mixed Gaussian and Plane Waves Approach. Comput. Phys. Commun. 2005, 167 (2), 103-128.

(72) Hutter, J.; Iannuzzi, M.; Schiffmann, F.; VandeVondele, J. Cp2k: Atomistic Simulations of Condensed Matter Systems. Wiley Interdiscip. Rev. Comput. Mol. Sci. 2014, 4 (1), 15-25.

(73) Byrd, R. H.; Lu, P.; Nocedal, J.; Zhu, C. A Limited Memory Algorithm for Bound Constrained Optimization. SIAM J. Sci. Comput. 1995, 16 (5), 1190-1208.

(74) Perdew, J. P.; Burke, K.; Ernzerhof, M. Generalized Gradient Approximation Made Simple. Phys. Rev. Lett. 1996, 77 (18), 38653868.

(75) Zhang, Y.; Yang, W. Comment on "Generalized Gradient Approximation Made Simple. Phys. Rev. Lett. 1998, 80 (4), 890-890.

(76) Grimme, S.; Antony, J.; Ehrlich, S.; Krieg, H. A Consistent and Accurate $\mathrm{Ab}$ Initio Parametrization of Density Functional Dispersion 
Correction (DFT-D) for the 94 Elements H-Pu. J. Chem. Phys. 2010, 132 (15), 154104.

(77) VandeVondele, J.; Hutter, J. Gaussian Basis Sets for Accurate Calculations on Molecular Systems in Gas and Condensed Phases. J. Chem. Phys. 2007, 127 (11), 114105.

(78) Goedecker, S.; Teter, M.; Hutter, J. Separable Dual-Space Gaussian Pseudopotentials. Phys. Rev. B: Condens. Matter Mater. Phys. 1996, 54 (3), 1703-1710.

(79) Hartwigsen, C.; Goedecker, S.; Hutter, J. Relativistic Separable Dual-Space Gaussian Pseudopotentials from H to Rn. Phys. Rev. B: Condens. Matter Mater. Phys. 1998, 58 (7), 3641-3662.

(80) Krack, M. Pseudopotentials for $\mathrm{H}$ to $\mathrm{Kr}$ Optimized for Gradient-Corrected Exchange-Correlation Functionals. Theor. Chem. Acc. 2005, 114 (1-3), 145-152.

(81) Bussi, G.; Donadio, D.; Parrinello, M. Canonical Sampling through Velocity Rescaling. J. Chem. Phys. 2007, 126 (1), 014101.

(82) Lu, Z.; Karakoti, A.; Velarde, L.; Wang, W.; Yang, P.; Thevuthasan, S.; Wang, H. Dissociative Binding of Carboxylic Acid Ligand on Nanoceria Surface in Aqueous Solution: A Joint In Situ Spectroscopic Characterization and First-Principles Study. J. Phys. Chem. C 2013, 117 (46), 24329-24338.

(83) Lu, R.; Gan, W.; Wu, B.; Chen, H.; Wang, H. Vibrational Polarization Spectroscopy of $\mathrm{CH}$ Stretching Modes of the Methylene Group at the Vapor/Liquid Interfaces with Sum Frequency Generation. J. Phys. Chem. B 2004, 108 (22), 7297-7306.

(84) Ohto, T.; Usui, K.; Hasegawa, T.; Bonn, M.; Nagata, Y. Toward $\mathrm{Ab}$ Initio Molecular Dynamics Modeling for Sum-Frequency Generation Spectra; an Efficient Algorithm Based on Surface-Specific Velocity-Velocity Correlation Function. J. Chem. Phys. 2015, 143 (12), 124702.

(85) Mukamel, S. Principles of Nonlinear Optical Spectroscopy; Oxford series in optical and imaging sciences; Oxford University Press, 1999.

(86) Liu, J.; Miller, W. H.; Paesani, F.; Zhang, W.; Case, D. A. Quantum Dynamical Effects in Liquid Water: A Semiclassical Study on the Diffusion and the Infrared Absorption Spectrum. J. Chem. Phys. 2009, 131 (16), 164509.

(87) Berens, P. H.; Wilson, K. R. Molecular Dynamics and Spectra. I. Diatomic Rotation and Vibration. J. Chem. Phys. 1981, 74 (9), $4872-4882$.

(88) Corcelli, S. A.; Skinner, J. L. Infrared and Raman Line Shapes of Dilute HOD in Liquid H $2 \mathrm{O}$ and D 20 from 10 to $90^{\circ} \mathrm{C}$. J. Phys. Chem. A 2005, 109 (28), 6154-6165.

(89) Auer, B. M.; Skinner, J. L. IR and Raman Spectra of Liquid Water: Theory and Interpretation. J. Chem. Phys. 2008, 128 (22), 224511.

(90) Willard, A. P.; Chandler, D. Instantaneous Liquid Interfaces. J. Phys. Chem. B 2010, 114 (5), 1954-1958.

(91) Serva, A.; Pezzotti, S.; Bougueroua, S.; Galimberti, D. R.; Gaigeot, M.-P. Combining Ab-Initio and Classical Molecular Dynamics Simulations to Unravel the Structure of the 2D-HBNetwork at the Air-Water Interface. J. Mol. Struct. 2018, 1165, 71-78.

(92) Pezzotti, S.; Galimberti, D. R.; Shen, Y. R.; Gaigeot, M.-P. Structural Definition of the BIL and DL: A New Universal Methodology to Rationalize Non-Linear $\chi(2)(\omega)$ SFG Signals at Charged Interfaces, Including $\chi(3)(\omega)$ Contributions. Phys. Chem. Chem. Phys. 2018, 20 (7), 5190-5199.

(93) Auer, B.; Kumar, R.; Schmidt, J. R.; Skinner, J. L. Hydrogen Bonding and Raman, IR, and 2D-IR Spectroscopy of Dilute HOD in Liquid D2O. Proc. Natl. Acad. Sci. U. S. A. 2007, 104 (36), 1421514220 .

(94) Richmond, G. L. Molecular Bonding and Interactions at Aqueous Surfaces as Probed by Vibrational Sum Frequency Spectroscopy. Chem. Rev. 2002, 102 (8), 2693-2724.

(95) Joutsuka, T.; Hirano, T.; Sprik, M.; Morita, A. Effects of ThirdOrder Susceptibility in Sum Frequency Generation Spectra: A Molecular Dynamics Study in Liquid Water. Phys. Chem. Chem. Phys. 2018, 20 (5), 3040-3053.
(96) Ohno, P. E.; Wang, H.; Paesani, F.; Skinner, J. L.; Geiger, F. M. Second-Order Vibrational Lineshapes from the Air/Water Interface. J. Phys. Chem. A 2018, 122 (18), 4457-4464.

(97) Morita, A.; Hynes, J. T. A Theoretical Analysis of the Sum Frequency Generation Spectrum of the Water Surface. II. TimeDependent Approach. J. Phys. Chem. B 2002, 106 (3), 673-685.

(98) Nihonyanagi, S.; Mondal, J. A.; Yamaguchi, S.; Tahara, T. Structure and Dynamics of Interfacial Water Studied by HeterodyneDetected Vibrational Sum-Frequency Generation. Annu. Rev. Phys. Chem. 2013, 64 (1), 579-603.

(99) Ji, N.; Ostroverkhov, V.; Tian, C. S.; Shen, Y. R. Characterization of Vibrational Resonances of Water-Vapor Interfaces by Phase-Sensitive Sum-Frequency Spectroscopy. Phys. Rev. Lett. 2008, 100 (9), 096102.

(100) Ohto, T.; Tada, H.; Nagata, Y. Structure and Dynamics of Water at Water-Graphene and Water-Hexagonal Boron-Nitride Sheet Interfaces Revealed by Ab Initio Sum-Frequency Generation Spectroscopy. Phys. Chem. Chem. Phys. 2018, 20 (18), 12979-12985.

(101) Wen, Y.-C.; Zha, S.; Tian, C.; Shen, Y. R. Surface PH and Ion Affinity at the Alcohol-Monolayer/Water Interface Studied by SumFrequency Spectroscopy. J. Phys. Chem. C 2016, 120 (28), 1522415229.

(102) Reddy, S. K.; Thiraux, R.; Wellen Rudd, B. A.; Lin, L.; Adel, T.; Joutsuka, T.; Geiger, F. M.; Allen, H. C.; Morita, A.; Paesani, F. Bulk Contributions Modulate the Sum-Frequency Generation Spectra of Water on Model Sea-Spray Aerosols. Chem. 2018, 4 (7), 16291644.

(103) Moberg, D. R.; Li, Q.; Reddy, S. K.; Paesani, F. Water Structure at the Interface of Alcohol Monolayers as Determined by Molecular Dynamics Simulations and Computational Vibrational Sum-Frequency Generation Spectroscopy. J. Chem. Phys. 2019, 150 (3), 034701.

(104) Lu, R.; Gan, W.; Wu, B.; Zhang, Z.; Guo, Y.; Wang, H. C-H Stretching Vibrations of Methyl, Methylene and Methine Groups at the Vapor/Alcohol $(\mathrm{n}=1-8)$ Interfaces. J. Phys. Chem. B 2005, 109 (29), 14118-14129.

(105) Xu, J.; Chen, M.; Zhang, C.; Wu, X. First-Principles Study of the Infrared Spectrum in Liquid Water from a Systematically Improved Description of H-Bond Network. Phys. Rev. B: Condens. Matter Mater. Phys. 2019, 99 (20), 205123.

(106) Tuladhar, A.; Dewan, S.; Kubicki, J. D.; Borguet, E. Spectroscopy and Ultrafast Vibrational Dynamics of Strongly Hydrogen Bonded $\mathrm{OH}$ Species at the $\alpha$-Al $2 \mathrm{O} 3(11 \overline{2} 0) / \mathrm{H} 2 \mathrm{O}$ Interface. J. Phys. Chem. C 2016, 120 (29), 16153-16161.

(107) Sulpizi, M.; Gaigeot, M.-P.; Sprik, M. The Silica-Water Interface: How the Silanols Determine the Surface Acidity and Modulate the Water Properties. J. Chem. Theory Comput. 2012, 8 (3), 1037-1047.

(108) Gaigeot, M.-P.; Sprik, M.; Sulpizi, M. Oxide/Water Interfaces: How the Surface Chemistry Modifies Interfacial Water Properties. J. Phys.: Condens. Matter 2012, 24 (12), 124106.

(109) Ishiyama, T.; Imamura, T.; Morita, A. Theoretical Studies of Structures and Vibrational Sum Frequency Generation Spectra at Aqueous Interfaces. Chem. Rev. 2014, 114 (17), 8447-8470. 